\title{
Regularity of probability laws by using an interpolation method
}

\author{
VLAD BALLY* \\ LuCia CARAMELlino ${ }^{\dagger}$
}

October 15, 2018

\begin{abstract}
We study the problem of the existence and regularity of a probability density in an abstract framework based on a "balancing" with approximating absolutely continuous laws. Typically, the absolutely continuous property for the approximating laws can be proved by standard techniques from Malliavin calculus whereas for the law of interest no Malliavin integration by parts formulas are available. Our results are strongly based on the use of suitable Hermite polynomial series expansions and can be merged into the theory of interpolation spaces. We then apply the results to the solution to a stochastic differential equation with a local Hörmander condition or to the solution to the stochastic heat equation, in both cases under weak conditions on the coefficients relaxing the standard Lipschitz or Hölder continuity requests.
\end{abstract}

Keywords: Young functions, Orlicz spaces, Hermite polynomials, interpolation spaces, Malliavin integration by parts formulas.

2010 MSC: 60H07, 46B70, 60H30.

\section{Contents}

1 Introduction

2 Criterion for the regularity of a probability law

2.1 Notation and main results . . . . . . . . . . . . . . . . . . 4

2.2 Hermite expansions and density estimates . . . . . . . . . . . . . . . . . . . . . . . . . . . . . .

2.3 Integration by parts formulas . . . . . . . . . . . . . . . . . 16

3 Interpolation spaces 20

3.1 Distribution spaces . . . . . . . . . . . . . . . . . . . . . . . . . . . . . . . . . . .

3.2 Negative Sobolev spaces . . . . . . . . . . . . . . . . . . 23

*Laboratoire d'Analyse et de Mathématiques Appliquées, UMR 8050, Université Paris-Est Marnela-Vallée, 5 Bld Descartes, Champs-sur-Marne, 77454 Marne-la-Vallée Cedex 2, France. Email: bally@univ-mlv.fr

†Dipartimento di Matematica, Università di Roma - Tor Vergata, Via della Ricerca Scientifica 1, I-00133 Roma, Italy. Email: caramel1@mat.uniroma2.it 
4 Diffusion processes 24

4.1 A local ellipticity condition . . . . . . . . . . . . . . 25

4.2 A local Hörmander condition . . . . . . . . . . . . . . . . . . 28

5 Stochastic heat equation 32

6 Appendix 36

6.1 Super kernels . . . . . . . . . . . . . . . . . . . 36

6.2 Norms . . . . . . . . . . . . . . . . . . . . . . . . . . . . . 39

References

\section{Introduction}

P. Malliavin has built a stochastic differential calculus which allows one to prove integration by parts formulas of type $\mathbb{E}\left(\partial_{\alpha} \phi(F)\right)=\mathbb{E}\left(\phi(F) H_{\alpha}(F)\right)$ and used them in order to study the regularity of the law of $F$. Here $F$ is a functional on the Wiener space. Roughly speaking the strategy is the following: on takes a sequence of simple functionals $F_{n} \rightarrow F$, defines the differential operators $D F_{n}$ and then, if $F \in D o m D$, defines $D F=\lim _{n} D F_{n}$. And this infinite dimensional differential calculus allows one to prove the integration by parts formulas. But one may proceed in a different way: using the finite dimensional calculus associated to simple functionals one proves $\mathbb{E}\left(\partial_{\alpha} \phi\left(F_{n}\right)\right)=\mathbb{E}\left(\phi\left(F_{n}\right) H_{\alpha}\left(F_{n}\right)\right)$, use it in order to get estimates of the Fourier transform and then pass to the limit. Of course, if everything works well when passing to the limit, this is more or less the same. But the interesting point is that one may use this strategy even if $\mathbb{E}\left(\phi\left(F_{n}\right) H_{\alpha}\left(F_{n}\right)\right)$ does not converge, so for $F \notin D o m D$. In fact, consider a random variable $F$ and a sequence of functionals $F_{n}, n \in \mathbb{N}$ such that $\mathbb{E}\left|F-F_{n}\right| \rightarrow 0$ and suppose that some integration by parts formulas hold for each $F_{n}$ (does not matter how one obtains them). Then one can proceed as follows (for simplicity we consider the one dimensional case). Let $\widehat{p}_{F_{n}}(\xi)=\mathbb{E}\left(e^{i \xi F_{n}}\right)$ be the Fourier transform of $F_{n}$. Since $\partial_{x}^{k} e^{i \xi F_{n}}=(i \xi)^{k} e^{i \xi F_{n}}$ one may use $k$ integration by parts and obtains

$$
\widehat{p}_{F_{n}}(\xi)=\frac{1}{(i \xi)^{k}} \mathbb{E}\left(\partial_{x}^{k} e^{i \xi F_{n}}\right)=\frac{1}{(i \xi)^{k}} \mathbb{E}\left(e^{i \xi F_{n}} H_{k}\left(F_{n}\right)\right) .
$$

Then one writes

$$
\begin{aligned}
\left|\widehat{p}_{F}(\xi)\right| & =\left|\widehat{p}_{F}(\xi)-\widehat{p}_{F_{n}}(\xi)\right|+\frac{1}{|\xi|^{k}}\left|\mathbb{E}\left(e^{i \xi F_{n}} H_{k}\left(F_{n}\right)\right)\right| \\
& \leq|\xi| \mathbb{E}\left|F-F_{n}\right|+\frac{1}{|\xi|^{k}} \mathbb{E}\left|H_{k}\left(F_{n}\right)\right| .
\end{aligned}
$$

So if one succeeds to get a good balance between $\mathbb{E}\left|F-F_{n}\right| \downarrow 0$ and $\mathbb{E}\left|H_{k}\left(F_{n}\right)\right| \uparrow \infty$ one may obtain good estimates of $\left|\widehat{p}_{F}(\xi)\right|$ and this implies the regularity of the law of $F$.

This argument originates from [12] and has been used in several recent papers: see [2, [3], [4], 9] and [14. Notice however that this method depends on the dimension: the weaker condition which gives a density of the law of $F$ is $\int_{\mathbb{R}^{d}}\left|\widehat{p}_{X_{T}}(\xi)\right|^{2} d x<\infty$ and of course, 
this depends on $d$. Let us give a simple but significant example (see [14]). Consider a $d$ dimensional diffusion process $d X_{t}=\sum_{j=1}^{N} \sigma_{j}\left(X_{t}\right) d W_{t}^{j}+b\left(X_{t}\right) d t$. In [14] one assumes that the coefficients $\sigma_{j}$ and $b$ are Hölder continuous of order $h>0$ and tries to prove that the law of $X_{T}$ is absolutely continuous. One takes $\delta>0$ and defines $X_{t}^{\delta}$ to be equal to $X_{t}$ for $t \leq T-\delta$ and $X_{t}^{\delta}=X_{T-\delta}+\sum_{j=1}^{N} \sigma_{j}\left(X_{T-\delta}\right)\left(W_{t}^{j}-W_{T-\delta}^{j}\right)$ for $t \geq T-\delta$. It is easy to see that $\mathbb{E}\left|X_{T}-X_{T}^{\delta}\right| \leq C \delta^{\frac{1}{2}(1+h)}$. On the other hand if $\sigma \sigma^{*} \geq c$ then conditionally to $X_{T-\delta}, X_{T}^{\delta}$ is a non degenerated Gaussian random variable. Using elementary integration by parts one may prove that for every multi index $\alpha$ of length $k, \mathbb{E}\left(\partial_{\alpha} \phi\left(X_{T}^{\delta}\right)\right)=\mathbb{E}\left(\phi\left(X_{T}^{\delta}\right) H_{\alpha, \delta}\right)$ and $\mathbb{E}\left|H_{\alpha, \delta}\right| \leq C \delta^{-k / 2}$. Using (1.1)

$$
\left|\widehat{p}_{X_{T}}(\xi)\right| \leq|\xi| \mathbb{E}\left|X_{T}-X_{T}^{\delta}\right|+\frac{1}{|\xi|^{k}} \mathbb{E}\left|H_{\alpha, \delta}\right| \leq C|\xi| \delta^{\frac{1}{2}(1+h)}+\frac{C}{|\xi|^{k} \delta^{k / 2}} .
$$

We fix $\xi$ and we choose $\delta=|\xi|^{-2(k+1) /(k+1+h)}$ in order to optimize the above relation. Then

$$
\left|\widehat{p}_{X_{T}}(\xi)\right| \leq C|\xi|^{-\frac{h k}{1+h+k}} .
$$

If $d=1$ and $h>\frac{1}{2}$ one may choose $k$ sufficiently large in order to get $\frac{h k}{1+h+k}>\frac{1}{2}$ and so $\int_{\mathbb{R}^{d}}\left|\widehat{p}_{X_{T}}(\xi)\right|^{2} d x<\infty$. But for $d \geq 2$, even if $h=1$, one fails to prove that the above integral is finite. So this approach is successful just for $d=1$ and $h>\frac{1}{2}$.

The aim of this paper is to obtain a more performing balance which essentially does not depend on the dimension. The main results are Theorem 2.4 in Section 2.1 and Theorem 2.17 in Section 2.3. Our estimates are based on a development in Hermite series (instead of the Fourier transform) presented in Section 2.2. In this framework we use a powerful result concerning the regularity of a mixture of Hermite functions - see Theorem 2.8 in Section [2.2. This result has been proved in [11] in the one dimensional case then in [10] for the multi dimensional case. We use a variant given in [22] Corollary 2.3.

As an application of Theorem 2.4 we are able to improve the above mentioned result of [14] in the following way. For an open domain $D \subset \mathbb{R}^{d}$ we denote by $C_{\log }(D)$ the class of functions $f: D \rightarrow \mathbb{R}$ for which there exists $C, h>0$ such that $|f(x)-f(y)| \leq$ $C|\ln | x-\left.y\right|^{-h}$ for every $x, y \in D$. We fix $y_{0} \in \mathbb{R}^{d}$ and $r>0$ and we suppose that the coefficients of the SDE presented above verify $\sigma_{j} \in C_{\log }\left(B_{r}\left(y_{0}\right)\right), j=1, \ldots, N$ (the drift coefficient $b$ is just measurable with linear growth). We also assume that $\sigma \sigma^{*}\left(y_{0}\right)>0$. Finally we consider an open domain $\Gamma \subset \mathbb{R}^{d}$ and we denote by $\tau$ the exit time from $\Gamma$. Our result (see Theorem 4.1) says that for $y_{0} \in \Gamma$ which verifies the above hypothesis the law of $X_{T \wedge \tau}$ is absolutely continuous with respect to the Lebesgue measure in a neighborhood of $y_{0}$. And this is true for any dimension $d$ and every $h>0$. Notice that even if the coefficients are smooth one cannot use directly the Malliavin calculus because, due to the stopping time $\tau, X_{T \wedge \tau}$ is not differentiable in Malliavin sense. In [7] it is given a variant of Malliavin calculus which permits to handle SDE's with boundary conditions - but there the coefficients are smooth (while here they are just in $C_{\log }\left(B_{r}\left(y_{0}\right)\right)$ ). Finally in Section 5 we prove a similar absolute continuity result for solutions of the stochastic heat equation. We mention also that recently Debussche and Romito [8] introduced an alternative approach, based on Besov space techniques, which enables one to prove the above result for Hölder continuous coefficients. This technique has already been used by Fournier [13] in order to study the regularity of the 3-dimensional Boltzman equation. 
After having done the work concerning probability measures we realized that this fits in the more general theory of interpolation spaces and extends to distributions (we thank to D. Elworthy who remarked this). In this framework our criterion ensures that a given distribution (in particular a probability measure) belongs to a certain interpolation space between a distribution space and a weighted Sobolev space. And the work done in the paper consists in proving that such an interpolation space is in between two Sobolev spaces. This gives the regularity result. There already exist a certain number of results concerning interpolation between negative Sobolev spaces and Sobolev spaces. But our result does not seem to fit in this framework. The reason is that when working with Sobolev spaces one employs $L^{p}$ norms while the distribution space that we consider is defined in terms of $L^{\infty}$ norms. And the case of $L^{\infty}$ norms appears as a limit case in interpolation theory and is more delicate to treat (see Triebel [27] or Bennet and Sharepley [6]).

The paper is organized as follows. In Section 2.1 we state the main result which is a criterion of regularity for general finite measures. We prove it in Section 2.2, and in Section 2.3 we give an alternative regularity criterion using integration by parts formulas. In Section 3 we discuss the link with interpolation spaces. In Section 4 we give two examples. The first one concerns diffusion processes with coefficients in $C_{\log }(D)$ under an ellipticity assumption (the example presented above). It turns out that in this case one does not need to use integration by parts: the analysis relies on the explicit Gaussian density. The second example concerns diffusions with more regular coefficients which verify a local Hörmander condition. In this case the integration by parts formula from Malliavin calculus is used. Finally, in Section 5 we prove a regularity result for the stochastic heat equation introduced by Walsh [28]. This considerably improves a previous result of Bally and Pardoux [5]. In the Appendix we discuss and prove some properties related to interpolation spaces.

\section{Criterion for the regularity of a probability law}

\subsection{Notation and main results}

We work on $\mathbb{R}^{d}$ and we denote by $\mathcal{M}$ the set of the finite signed measures on $\mathbb{R}^{d}$ with the Borel $\sigma$ algebra. Moreover $\mathcal{M}_{a} \subset \mathcal{M}$ is the set of the measures which are absolutely continuous with respect to the Lebesgue measure. For $\mu \in \mathcal{M}_{a}$ we denote by $p_{\mu}$ the density of $\mu$ with respect to the Lebesgue measure. And for a measure $\mu \in \mathcal{M}$ we denote by $L_{\mu}^{p}$ the space of the measurable functions $f: \mathbb{R}^{d} \rightarrow \mathbb{R}$ such that $\int|f|^{p} d \mu<\infty$. For $f \in L_{\mu}^{1}$ we denote $f \mu$ the measure $(f \mu)(A)=\int_{A} f d \mu$. For a bounded function $\phi: \mathbb{R}^{d} \rightarrow \mathbb{R}$ we denote $\mu * \phi$ the measure defined by $\int f d \mu * \phi=\int f * \phi d \mu=\iint \phi(x-y) f(y) d y d \mu(x)$. Then $\mu * \phi \in \mathcal{M}_{a}$ and $p_{\mu * \phi}(x)=\int \phi(x-y) d \mu(y)$.

We denote by $\alpha=\left(\alpha_{1}, \ldots, \alpha_{d}\right) \in \mathbb{N}^{d}$ a multi index and we put $|\alpha|=\sum_{i=1}^{d} \alpha_{i}$. Here $\mathbb{N}=\{0,1,2, \ldots\}$ are the non negative integers and we put $\mathbb{N}_{*}=\mathbb{N} \backslash\{0\}$. For a multi index $\alpha$ with $|\alpha|=k$ we denote $\partial_{\alpha}$ the corresponding derivative that is $\partial_{x_{1}}^{\alpha_{1}} \ldots \partial_{x_{d}}^{\alpha_{d}}$ with the convention that $\partial_{x_{i}}^{\alpha_{i}} f=f$ if $\alpha_{i}=0$. In particular if $\alpha$ is the null multi index then $\partial_{\alpha} f=f$. We denote by $\|f\|_{p}=\left(\int|f(x)|^{p} d x\right)^{1 / p}, p \geq 1$ and $\|f\|_{\infty}=\sup _{x \in \mathbb{R}^{d}}|f(x)|$. Then $L^{p}=\{f$ : 
$\left.\|f\|_{p}<\infty\right\}$ are the standard $L^{p}$ spaces with respect to the Lebesgue measure.

In the following we will work in Orlicz spaces so we briefly recall the notation and the results we will use (we refer to [16]). A function $e: \mathbb{R} \rightarrow \mathbb{R}_{+}$is a Young function if it is symmetric, strictly convex, non negative and $e(0)=0$. In the following we will consider a Young function which has the two supplementary properties:

$$
\begin{aligned}
& \text { i) there exists } \lambda>0 \text { such that } e(2 s) \leq \lambda e(s), \\
& \text { ii) } s \mapsto \frac{e(s)}{s} \text { is non decreasing. }
\end{aligned}
$$

The property $i$ ) is known as the $\Delta_{2}$ condition or doubling condition (see [16]). Through the whole paper we work with Young functions which satisfy (2.1). We denote by $\mathcal{E}$ the space of these functions.

For $e \in \mathcal{E}$ and $f: \mathbb{R}^{d} \rightarrow \mathbb{R}$, we define the norm

$$
\|f\|_{(e)}=\inf \left\{c>0: \int e\left(\frac{1}{c} f(x)\right) d x \leq 1\right\} .
$$

This is the so called Luxembourg norm which is equivalent to the Orlicz norm (see [16] p 227 Th 7.5.4). It is convenient for us to work with this norm (instead of the Orlicz norm). The space $L^{e}=\left\{f:\|f\|_{(e)}<\infty\right\}$ is the Orlicz space with respect to the Lebesgue measure. Notice that if we take $e_{p}(x)=|x|^{p}, p>1$, then $\|f\|_{\left(e_{p}\right)}$ is the usual $L^{p}$ norm and the corresponding Orlicz space is the standard $L^{p}$ space. Another example is given at the end of this section.

Remark 2.1 Let $u_{l}(x)=(1+|x|)^{-l}$. As a consequence of (2.1) ii), for every $l>d$ one has

$$
\left\|u_{l}\right\|_{e} \leq\left(e(1)\left\|u_{l}\right\|_{1}\right) \vee 1<\infty .
$$

Indeed (2.1) ii) implies that for $t \leq 1$ one has $e(t) \leq e(1) t$. For $c>\left(e(1)\left\|u_{l}\right\|_{1}\right) \vee 1$ one has $\frac{1}{c} u_{l}(x) \leq u_{l}(x) \leq 1$ so that

$$
\int e\left(\frac{1}{c} u_{l}(x)\right) d x \leq \frac{e(1)}{c} \int u_{l}(x) d x=\frac{e(1)}{c}\left\|u_{l}\right\|_{1} \leq 1 .
$$

One defines the conjugate of $e$ by $e_{*}(s)=\inf \{s t-e(t): t \in \mathbb{R}\}$ and this is also a Young function so the corresponding Luxembourg norm $\|f\|_{\left(e_{*}\right)}$ is given by (2.2) with $e$ replaced by $e_{*}$. Then we have the following Hölder inequality

$$
\left|\int f g(x) d x\right| \leq 2\|f\|_{(e)}\|g\|_{\left(e_{*}\right)} .
$$

See [16] p 215 Th 7.2.1 (the factor 2 does not appear in Th 7.2.1 but there in the right hand side of the inequality one has the Orlicz norm of $g$. Using the equivalence between the Orlicz and the Luxembourg norm we replace the Orlicz norm by $\left.2\|g\|_{\left(e_{*}\right)}\right)$.

If $e$ satisfies the $\Delta_{2}$ condition (that is (2.1) i) above) then $L^{e}$ is reflexive (see [16] p $234 \mathrm{Th}$ 7.7.1). In particular, in this case, any bounded subset of $L^{e}$ is weakly relative compact. We also define $e^{-1}(a)=\sup \{c: e(c) \leq a\}$ and

$$
\phi_{e}(r)=\frac{1}{e^{-1}\left(\frac{1}{r}\right)} \quad \text { and } \quad \beta_{e}(R)=\frac{R}{e^{-1}(R)}=R \phi_{e}\left(\frac{1}{R}\right) \text {. }
$$


Remark 2.2 The function $\phi_{e}$ is the "fundamental function" of $L^{e}$ equipped with the Luxembourg norm (see [6] Lemma $8.17 \mathrm{pg}$ 276). In particular $\frac{1}{r} \phi_{e}(r)$ is decreasing (see [6] Corollary 5.2 $\mathrm{pg}$ 67). It follows that $\beta_{e}$ is increasing. For the sake of completeness we give here the argument. Indeed, if $a>1$ then $e(a x) \geq a e(x)$ so that ax $\geq e^{-1}(a e(x))$. Taking $y=e(x)$ we obtain a $e^{-1}(y) \geq e^{-1}($ ay $)$ which gives

$$
\beta_{e}(a y)=\frac{a y}{e^{-1}(a y)} \geq \frac{a y}{a e^{-1}(y)}=\beta_{e}(y)
$$

We introduce now the norms

$$
\|f\|_{k,(e)}=\sum_{0 \leq|\alpha| \leq k}\left\|\partial_{\alpha} f\right\|_{(e)} \quad \text { and } \quad\|f\|_{k, \infty}=\sum_{0 \leq|\alpha| \leq k}\left\|\partial_{\alpha} f\right\|_{\infty}
$$

We denote

$$
W^{k, e}=\left\{f:\|f\|_{k,(e)}<\infty\right\} \quad \text { and } \quad W^{k, \infty}=\left\{f:\|f\|_{k, \infty}<\infty\right\} .
$$

For a multi index $\gamma$ we denote $x^{\gamma}=\prod_{i=1}^{d} x_{i}^{\gamma_{i}}$ and for two multi indexes $\alpha, \gamma$ we denote $f_{\gamma, \alpha}$ the function $f_{\alpha, \gamma}(x)=x^{\gamma} \partial_{\alpha} f(x)$. Then we consider the norm

$$
\|f\|_{k, l,(e)}=\sum_{0 \leq|\gamma| \leq l} \sum_{0 \leq|\alpha| \leq k}\left\|f_{\gamma, \alpha}\right\|_{(e)} \quad \text { and } \quad W^{k, l, e}=\left\{f:\|f\|_{k, l,(e)}<\infty\right\} .
$$

We stress that in $\|\cdot\|_{k, l,(e)}$ the first index $k$ is related to the order of the derivatives which are involved while the second index $l$ is connected to the power of the polynomial multiplying the function and its derivatives up to order $k$.

We consider the following distances between two measures $\mu, \nu \in \mathcal{M}$. For $k \in \mathbb{N}$

$$
d_{k}(\mu, \nu)=\sup \left\{\left|\int \phi d \mu-\int \phi d \nu\right|: \phi \in C^{\infty}\left(\mathbb{R}^{d}\right),\|\phi\|_{k, \infty} \leq 1\right\} .
$$

Notice that $d_{0}$ is the total variation distance and $d_{1}$ is the bounded variation distance. The Wasserstein distance (which is more popular) is $d_{W}(\mu, \nu)=\sup \left\{\left|\int \phi d \mu-\int \phi d \nu\right|: \phi \in\right.$ $\left.C^{1}\left(\mathbb{R}^{d}\right),\|\nabla \phi\|_{\infty} \leq 1\right\}$ so $d_{1}(\mu, \nu) \leq d_{W}(\mu, \nu)$. It follows that all the results proved with respect to $d_{1}$ will be a fortiori true for $d_{W}$. The distances $d_{k}$ with $k \geq 2$ are less often used. We mention however that people working in approximation theory (for diffusion process for example - see [26] or [20]) use such distances in an implicit way: indeed, they study the speed of convergence of certain schemes but they are able to obtain their estimates for test functions $f \in C^{k}$ with $k$ sufficiently large - so $d_{k}$ comes on.

Let $q, k \in \mathbb{N}$ and $m \in \mathbb{N}_{*}$. For $\mu \in \mathcal{M}$ and for a sequence $\mu_{n} \in \mathcal{M}_{a}, n \in \mathbb{N}$ we define

$$
\pi_{q, k, m, e}\left(\mu,\left(\mu_{n}\right)_{n}\right)=\sum_{n=0}^{\infty} 2^{n(q+k)} \beta_{e}\left(2^{n d}\right) d_{k}\left(\mu, \mu_{n}\right)+\sum_{n=0}^{\infty} \frac{1}{2^{2 n m}}\left\|p_{\mu_{n}}\right\|_{2 m+q, 2 m,(e)} .
$$

Moreover we define

$$
\rho_{q, k, m, e}(\mu)=\inf \pi_{q, k, m, e}\left(\mu,\left(\mu_{n}\right)_{n}\right)
$$


with the infimum taken over all the sequences $\mu_{n} \in \mathcal{M}_{a}, n \in \mathbb{N}$. We define

$$
\mathcal{S}_{q, k, m, e}=\left\{\mu \in \mathcal{M}: \rho_{q, k, m, e}(\mu)<\infty\right\} .
$$

It is easy to check that $\rho_{q, k, m, e}$ is a norm on $\mathcal{S}_{q, k, m, e}$.

The main result in this section is the following.

Theorem 2.3 Let $q, k \in \mathbb{N}, m \in \mathbb{N}_{*}$ and let $e \in \mathcal{E}$.

i) Take $q=0$. Then

$$
\mathcal{S}_{0, k, m, e} \subset L^{e}
$$

in the sense that if $\mu \in \mathcal{S}_{0, k, m, e}$ then $\mu$ is absolutely continuous and the density $p_{\mu}$ belongs to $L^{e}$. Moreover there exists a universal constant $C$ such that

$$
\left\|p_{\mu}\right\|_{L^{e}} \leq C \rho_{0, k, m, e}(\mu) \text {. }
$$

ii) Take $q \geq 1$. Then

$$
\mathcal{S}_{q, k, m, e} \subset W^{q, e} \quad \text { and } \quad\left\|p_{\mu}\right\|_{W^{q, e}} \leq C \rho_{q, k, m, e}(\mu), \quad \mu \in \mathcal{S}_{q, k, m, e} .
$$

The proof of this theorem is given at the end of Section 2.2.

It may be cumbersome to check that $\mu \in \mathcal{S}_{0, k, m, e}$ so we give a sufficient condition which seems to be more clear and easier to verify. We define

$$
M_{m, q, e}(R)=\left\{\mu \in \mathcal{M}_{a}:\left\|p_{\mu}\right\|_{2 m+q, 2 m,(e)} \leq R\right\} .
$$

For $a>1$ we denote

$$
L_{a}(R)=R(\ln R)^{a}
$$

and we consider the following hypothesis.

Hypothesis $H_{q}(k, m, e)$. For $q, k \in \mathbb{N}, m \in \mathbb{N}_{*}$ and $e \in \mathcal{E}$ there exists a $>1$ such that

$$
\varlimsup_{R \rightarrow \infty} \frac{L_{a}(R)^{1+\frac{k+q}{2 m}} \beta_{e}\left(L_{a}(R)^{\frac{d}{2 m}}\right)}{R} d_{k}\left(\mu, M_{m, q, e}(R)\right)<\infty .
$$

We define

$$
B_{q}(k, m, e)=\left\{\mu \in \mathcal{M}: H_{q}(k, m, e) \text { holds for } \mu\right\} .
$$

For $\alpha, \gamma \geq 0$ we denote by $\mathcal{E}_{\alpha, \gamma}$ the class of the Young functions $e \in \mathcal{E}$ such that

$$
0<\underline{\lim }_{R \rightarrow \infty} \frac{\beta_{e}(R)}{R^{\alpha}(\ln R)^{\gamma}} \leq \varlimsup_{R \rightarrow \infty} \frac{\beta_{e}(R)}{R^{\alpha}(\ln R)^{\gamma}}<\infty .
$$

The examples we have in mind fit in this class.

Our criterion is the following.

Theorem 2.4 i) Let $q, k \in \mathbb{N}, m \in \mathbb{N}_{*}$ and $e \in \mathcal{E}$. Then

$$
B_{q}(k, m, e) \subset \mathcal{S}_{q, k, m, e} \subset W^{q, e} .
$$

ii) Suppose that $e \in \mathcal{E}_{\alpha, \gamma}$ with $0 \leq \alpha<\frac{2 m+k+q}{d(2 m-1)}$ and $\gamma \geq 0$. Then

$$
W^{q+1,2 m, e} \subset B_{q}(k, m, e) \subset \mathcal{S}_{q, k, m, e} \subset W^{q, e},
$$

the first inclusion holding for $m>d / 2$. 
Proof. i) The proof of $B_{q}(k, m, e) \subset \mathcal{S}_{q, k, m, e}$ is given in Lemma 6.6 and $\mathcal{S}_{q, k, m, e} \subset W^{q, e}$ is Theorem 2.3.

ii) The inclusion $W^{q+1,2 m, e} \subset B_{q}(k, m, e)$ is proved in Corollary 6.3,

Remark 2.5 The above criterion involves a lot of parameters and it is not easy to understand at a first glance which is the significance of each of them. So we try to give a first interpretation on their meaning. Our aim is to prove that the measure $\mu$ has a certain regularity and we want to do this by approximating it by some regular measures $\nu \in M_{m, q, e}(R)$. The first parameter which we fix is q. It represents the order of regularity that we hope to obtain. If $q=0$ this means that we want to prove that $\mu$ is just absolutely continuous with respect to the Lebesgue measure and the density is in some Orlicz space defined by e. For example if $e(t)=|t|^{p}$ this is the $L^{p}$ space. If $q \geq 1$ then our aim is to obtain more regularity, namely to prove that the density is in the Sobolev space of order $q$. The second parameter is $k$. It characterizes the distance in which we estimate the approximation error. Once $q, e, k$ are chosen it remains $m$. The choice of $m$ is different. For the other parameters the choice comes from our decision to treat a problem or another: they are involved in the definition of the problem we treat. On the contrary, $m$ is a free parameter which we choose for technical reasons: for example in some concrete situations it is suitable to chose $m$ very large - such that $\frac{1}{m} \leq \varepsilon$ for some $\varepsilon>0$ for example. In the following it will become clear that $m$ represents the number of integration by parts that we use.

The hypothesis $H_{q}(k, m, e)$ seems difficult to check because of the function $\beta_{e}$ which is involved there in. So we give two significant examples.

Example 1. Let $p>1$ and $e_{p}(t)=|t|^{p}$. The corresponding Orlicz space is the standard $L^{p}$ space. And we have $\beta_{e_{p}}(t)=|t|^{1 / p_{*}}$ with $p_{*}$ the conjugate of $p$. So $e_{p} \in \mathcal{E}_{1 / p_{*}, 0}$. Our hypothesis is:

$$
H_{q}\left(k, m, e_{p}\right): \quad \exists a>1 \text { s.t. } \varlimsup_{R \rightarrow \infty} R^{\frac{q+k+d / p_{*}}{2 m}}(\ln R)^{a\left(1+\frac{q+k+d / p_{*}}{2 m}\right)} d_{k}\left(\mu, M_{m, q, e}(R)\right)<\infty .
$$

In this hypothesis the dimensiond is still present. But its contribution is very small when $p$ is close to one. Then Theorem 2.4 reads as follows. If $\mu$ satisfies $H_{q}\left(k, m, e_{p}\right)$ then $\mu(d x)=f(x) d x$ with $f \in W^{q, 2 m, p}$. Moreover, if $p_{*}>\frac{d(2 m-1)}{2 m+k+q}$ and $f \in W^{q+1, p}$ then the measure $\mu(d x)=f(x) d x$ satisfies $H_{q}\left(k, m, e_{p}\right)$.

Example 2. Set $e_{\log }(t)=(1+|t|) \ln (1+|t|)$. It is easy to check that $e_{\log }$ is a Young function which satisfies the property $\Delta_{2}$. The corresponding Orlicz space is the so called LlogL space of Zigmund, i.e the spaces of the functions $f$ such that $\int|f(x)| \ln ^{+}|f(x)| d x<\infty$ (see [6]). Let us check that $e_{\log } \in \mathcal{E}_{0,1}$. We denote $e_{a}(t)=$ at $\ln ($ at $)$ and we notice that for $t$ large we have $e_{1}(t) \leq e_{\log }(t) \leq e_{2}(t)$ (in particular $L^{e_{\log }}$ is the space of the functions which have finite entropy) Then $e_{2}^{-1}(t) \leq e_{\log }^{-1}(t) \leq e_{1}^{-1}(t)$ so that

$$
\frac{t}{e_{1}^{-1}(t)} \leq \beta_{e_{\log }}(t) \leq \frac{t}{e_{2}^{-1}(t)}
$$

Using the change of variable $R=e_{a}(t)$ one obtains

$$
\lim _{R \rightarrow \infty} \frac{R}{e_{1}^{-1}(R) \ln R}=\lim _{t \rightarrow \infty} \frac{e_{a}(t)}{t \ln e_{a}(t)}=a .
$$


This proves that

$$
1 \leq \underline{\lim }_{t \rightarrow \infty} \frac{\beta_{e_{\log }}(t)}{\ln t} \leq \varlimsup_{t \rightarrow \infty} \frac{\beta_{e_{\log }}(t)}{\ln t} \leq 2
$$

Our hypothesis is:

$$
H_{q}\left(k, m, e_{\log }\right): \quad \exists a>1 \text { s.t. } \varlimsup_{R \rightarrow \infty} R^{\frac{q+k}{2 m}}(\ln R)^{a\left(1+\frac{q+k}{2 m}\right)+1} d_{k}\left(\mu, M_{m, q, e_{\log }}(R)\right)<\infty .
$$

So the dimension $d$ does no more appear. Then Theorem 2.4 reads as follows. If $\mu$ satisfies $H_{q}\left(k, m, e_{\log }\right)$ then $\mu(d x)=p(x) d x$ with $p \in W^{q, e_{\log }}$. And if $p \in W^{q+1,2 m, e_{\log }}$ then the measure $\mu(d x)=p(x) d x$ satisfies $H_{q}\left(k, m, e_{\log }\right)$.

Remark 2.6 One may consider a further step and take $e_{\log \log }(t)=(1+|t|) \ln (1+\ln (1+$ $|t|))$. In this case $\beta_{e_{\log \log }}(t) \leq \ln \ln t$ but there will be no significant improvement: we obtain $(\ln R)^{a\left(1+\frac{q+k}{2 m}\right)} \ln \ln R$ instead of $(\ln R)^{a\left(1+\frac{q+k}{2 m}\right)+1}$.

Remark 2.7 Having in mind these examples we conclude that one may ask for two different questions. a) One may just want to prove that $\mu$ is absolutely continuous with respect to the Lebesgue measure. b) One wants to obtain estimates of the density in some given norm (associated to some Young function $e$ - for example in $L^{p}$ ). If the question

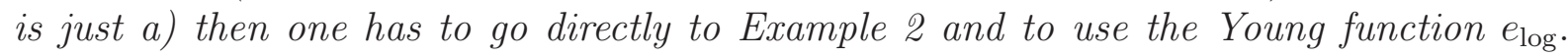
Because the hypothesis (2.12) is the minimal one (in our approach at least).

\subsection{Hermite expansions and density estimates}

We begin with a re-view of some basic properties of Hermite polynomials and functions. The Hermite polynomials on $\mathbb{R}$ are defined by

$$
H_{n}(t)=(-1)^{n} e^{t^{2}} \frac{d^{n}}{d t} e^{-t^{2}}, \quad n=0,1, \ldots
$$

They are orthogonal with respect to $e^{-t^{2}} d t$. We denote the $L^{2}$ normalized Hermite functions by

$$
h_{n}(t)=\left(2^{n} n ! \sqrt{\pi}\right)^{-1 / 2} H_{n}(t) e^{-t^{2} / 2}
$$

and we have

$$
\int_{R} h_{n}(t) h_{m}(t) d t=\left(2^{n} n ! \sqrt{\pi}\right)^{-1} \int_{R} H_{n}(t) H_{m}(t) e^{-t^{2}} d t=\delta_{n, m} .
$$

The Hermite functions form an orthonormal basis in $L^{2}(R)$. For a multi index $\alpha=$ $\left(\alpha_{1}, \ldots, \alpha_{d}\right) \in \mathbb{N}^{d}$ we define the $d$-dimensional Hermite function

$$
\mathcal{H}_{\alpha}(x):=\prod_{i=1}^{d} h_{\alpha_{i}}\left(x_{i}\right), \quad x=\left(x_{1}, \ldots, x_{d}\right)
$$

The $d$-dimensional Hermite functions form an orthonormal basis in $L^{2}\left(\mathbb{R}^{d}\right)$. This corresponds to the chaos decomposition in dimension $d$ (but the notation we gave above is 
slightly different from the one used in probability; see [21], 24] and [18, where Hermite polynomials are used. One may come back by a renormalization). The Hermite functions are the eigenvectors of the Hermite operator $D=-\Delta+|x|^{2}$ (with $\Delta$ the Laplace operator) and one has

$$
D \mathcal{H}_{\alpha}=(2|\alpha|+d) \mathcal{H}_{\alpha} \quad \text { with } \quad|\alpha|=\alpha_{1}+\ldots+\alpha_{d} .
$$

We denote $W_{n}=\operatorname{span}\left\{\mathcal{H}_{\alpha}:|\alpha|=n\right\}$ and we have $L^{2}\left(\mathbb{R}^{d}\right)=\oplus_{n=0}^{\infty} W_{n}$.

For a function $\Phi: \mathbb{R}^{d} \times \mathbb{R}^{d} \rightarrow \mathbb{R}$ and a function $f: \mathbb{R}^{d} \rightarrow \mathbb{R}$ we use the notation

$$
\Phi * f(x)=\int_{\mathbb{R}^{d}} \Phi(x, y) f(y) d y
$$

We denote by $J_{n}$ the orthogonal projection on $W_{n}$ and we have

$$
J_{n} v(x)=\mathcal{H}_{n} * v(x) \quad \text { with } \quad \mathcal{H}_{n}(x, y):=\sum_{|\alpha|=n} \mathcal{H}_{\alpha}(x) \mathcal{H}_{\alpha}(y)
$$

Moreover, we consider a function $a: \mathbb{R}_{+} \rightarrow \mathbb{R}$ whose support is included in $\left[\frac{1}{4}, 4\right]$ and we define

$$
\mathcal{H}_{n}^{a}(x, y)=\sum_{j=0}^{\infty} a\left(\frac{j}{4^{n}}\right) \mathcal{H}_{j}(x, y)=\sum_{j=4^{n-1}+1}^{4^{n+1}-1} a\left(\frac{j}{4^{n}}\right) \mathcal{H}_{j}(x, y), \quad x, y \in \mathbb{R}^{d}
$$

the last equality being a consequence of the support property of the function $a$.

The following estimate is a crucial point in our approach. It has been proved in [11], [10] and then in [22]. We refer to Corollary 2.3, inequality (2.17), in [22].

Theorem 2.8 Let $a: \mathbb{R}_{+} \rightarrow \mathbb{R}_{+}$be a non negative $C^{\infty}$ function with the support included in $\left[\frac{1}{4}, 4\right]$. We denote $\|a\|_{l}=\sum_{i=0}^{l} \sup _{t \geq 0}\left|a^{(i)}(t)\right|$. For every multi-index $\alpha$ and every $k \in \mathbb{N}$ there exists a constant $C_{k}$ (depending on $k, \alpha, d$ ) such that for every $n \in \mathbb{N}$ and every $x, y \in \mathbb{R}^{d}$

$$
\left|\frac{\partial^{|\alpha|}}{\partial x^{\alpha}} \mathcal{H}_{n}^{a}(x, y)\right| \leq C_{k}\|a\|_{k} \frac{2^{n(|\alpha|+d)}}{\left(1+2^{n}|x-y|\right)^{k}} .
$$

Following the ideas in 22 we consider a function $a: \mathbb{R}_{+} \rightarrow \mathbb{R}_{+}$of class $C_{b}^{\infty}$ with the support included in $\left[\frac{1}{4}, 4\right]$ and such that $a(t)+a(4 t)=1$ for $t \in\left[\frac{1}{4}, 1\right]$. We may construct $a$ in the following way: we take a function $a:[0,1] \rightarrow \mathbb{R}_{+}$with $a(t)=0$ for $t \leq \frac{1}{4}$ and $a(1)=1$. We may choose $a$ such that $a^{(l)}\left(\frac{1}{4}\right)=a^{(l)}(1-)=0$ for every $l \in \mathbb{N}$. Then we define $a(t)=1-a\left(\frac{t}{4}\right)$ for $t \in[1,4]$ and $a(t)=0$ for $t \geq 4$. This is the function we will use in the following. Notice that $a$ has the property:

$$
\sum_{n=0}^{\infty} a\left(\frac{t}{4^{n}}\right)=1 \quad \forall t \geq 1
$$

In order to check the above equality we fix $n_{t}$ such that $4^{n_{t}-1} \leq t<4^{n_{t}}$ and we notice that $a\left(\frac{t}{4^{n}}\right)=0$ if $n \notin\left\{n_{t}-1, n_{t}\right\}$. So $\sum_{n=0}^{\infty} a\left(\frac{t}{4^{n}}\right)=a(4 s)+a(s)=1$ with $s=t / 4^{n_{t}} \in\left[\frac{1}{4}, 1\right]$. In the following we fix a function $a$ and the constants in our estimates will depend on $\|a\|_{l}$ for some fixed $l$. Using this function we obtain the following representation formula: 
Proposition 2.9 For every $f \in L^{2}\left(\mathbb{R}^{d}\right)$

$$
f=\sum_{n=0}^{\infty} \mathcal{H}_{n}^{a} * f
$$

the series being convergent in $L^{2}\left(\mathbb{R}^{d}\right)$.

Proof. We fix $N$ and we denote

$$
S_{N}^{a}=\sum_{n=1}^{N} \mathcal{H}_{n}^{a} * f, \quad S_{N}=\sum_{j=1}^{4^{N}} \mathcal{H}_{j} * f \quad \text { and } \quad R_{N}^{a}=\sum_{j=4^{N}+1}^{4^{N+1}}\left(\mathcal{H}_{j} * f\right) a\left(\frac{j}{4^{N+1}}\right) .
$$

Let $j \leq 4^{N+1}$. For $n \geq N+2$ one has $a\left(\frac{j}{4^{n}}\right)=0$. So using (2.16) we obtain $\sum_{n=1}^{N} a\left(\frac{j}{4^{n}}\right)=$ $\sum_{n=1}^{\infty} a\left(\frac{j}{4^{n}}\right)-a\left(\frac{j}{4^{N+1}}\right)=1-a\left(\frac{j}{4^{N+1}}\right)$. And for $j \leq 4^{N}$ one has $a\left(\frac{j}{4^{N+1}}\right)=0$. It follows that

$$
\begin{aligned}
S_{N}^{a} & =\sum_{n=1}^{N} \sum_{j=0}^{\infty} a\left(\frac{j}{4^{n}}\right) \mathcal{H}_{j} * f=\sum_{n=1}^{N} \sum_{j=0}^{4^{N+1}} a\left(\frac{j}{4^{n}}\right) \mathcal{H}_{j} * f=\sum_{j=0}^{4^{N+1}}\left(\mathcal{H}_{j} * f\right) \sum_{n=1}^{N} a\left(\frac{j}{4^{n}}\right) \\
& =\sum_{j=0}^{4^{N+1}} \mathcal{H}_{j} * f-\sum_{j=4^{N}+1}^{4^{N+1}}\left(\mathcal{H}_{j} * f\right) a\left(\frac{j}{4^{N+1}}\right)=S_{N+1}-R_{N}^{a} .
\end{aligned}
$$

One has $S_{N} \rightarrow f$ in $L^{2}$ and $\left\|R_{N}^{a}\right\|_{2} \leq\|a\|_{\infty} \sum_{j=4^{N}+1}^{4^{N+1}}\left\|\mathcal{H}_{j} * f\right\|_{2} \rightarrow 0$ so the proof is completed.

We will need the following lemma concerning properties of the Luxembourg norms.

Lemma 2.10 Let $\rho \geq 0$ be a measurable function. Then for every measurable function $f$

$$
\|\rho * f\|_{(e)} \leq\|\rho\|_{1}\|f\|_{(e)} .
$$

Proof. Let $c=m\|f\|_{(e)}$ with $m=\|\rho\|_{1}=\int \rho(x-y) d y$. Since $e$ is convex we obtain

$$
\begin{aligned}
\int e\left(\frac{1}{c}(\rho * f)(x)\right) d x & =\int e\left(\int \frac{\rho(x-y)}{m} \times \frac{m}{c} f(y) d y\right) d x \\
& \leq \int d x \int \frac{\rho(x-y)}{m} \times e\left(\frac{m}{c} f(y)\right) d y \\
& =\int e\left(\frac{m}{c} f(y)\right) \int \frac{\rho(x-y)}{m} d x d y=\int e\left(\frac{m}{c} f(y)\right) d y \\
& =\int e\left(\frac{1}{\|f\|_{(e)}} f(y)\right) d y \leq 1
\end{aligned}
$$

and this means that $\|\rho * f\|_{(e)} \leq c=\|\rho\|_{1}\|f\|_{(e)}$. 
Lemma 2.11 Let $\rho_{n, p}(z)=\left(1+2^{n}|z|\right)^{-p}$ with $p>d$ and $e \in \mathcal{E}$. There exists a constant $C_{p}$ depending on $p$ and $d$ such that

$$
\left\|\rho_{n, p}\right\|_{(e)} \leq \frac{1}{e^{-1}\left(\frac{1}{C_{p}} 2^{n d}\right)}
$$

In particular, for $p=d+1$ there exists a constant $C$ depending on $d$ and on the doubling constant of e such that (with $\phi_{e}$ defined in 2.4)

$$
\left\|\rho_{n, d+1}\right\|_{(e)} \leq \frac{C}{e^{-1}\left(2^{n d}\right)}=C 2^{-n d} \beta_{e}\left(2^{n d}\right)=C \phi_{e}\left(\frac{1}{2^{n d}}\right)
$$

Proof. Let $c>0$. We pass in polar coordinates first and we use the change of variable $s=2^{n} r$ then and we obtain

$$
\begin{aligned}
\int_{\mathbb{R}^{d}} e\left(\frac{1}{c} \rho_{n, p}(z)\right) d z & =A_{d} \int_{0}^{\infty} r^{d-1} e\left(\frac{1}{c} \times \frac{1}{\left(1+2^{n} r\right)^{p}}\right) d r \\
& =2^{-n d} A_{d} \int_{0}^{\infty} s^{d-1} e\left(\frac{1}{c} \times \frac{1}{(1+s)^{p}}\right) d s
\end{aligned}
$$

where $A_{d}$ is the surface of the unit sphere in $\mathbb{R}^{d}$. Using the property (2.1) ii) we upper bound the above term by

$$
2^{-n d} e\left(\frac{1}{c}\right) A_{d} \int_{0}^{\infty} s^{d-1} \times \frac{1}{(1+s)^{p}} d s=C_{p} 2^{-n d} e\left(\frac{1}{c}\right) .
$$

In order to prove that $\left\|\rho_{n, p}\right\|_{(e)} \leq c$ we have to check that $\int_{\mathbb{R}^{d}} e\left(\frac{1}{c} \rho_{n, p}(z)\right) d z \leq 1$. In view of the above inequalities it suffices that $e\left(\frac{1}{c}\right) \leq 2^{\text {nd }} / C_{p}$ that is $c \geq 1 / e^{-1}\left(2^{n d} / C_{p}\right)$.

Proposition 2.12 Let $\alpha$ be a multi index.

i) There exists a universal constant $C$ (depending on $\alpha, d$ and $e$ ) such that

a) $\left\|\partial_{\alpha} \mathcal{H}_{n}^{a} * f\right\|_{(e)} \leq C\|a\|_{d+1} \times 2^{n|\alpha|}\|f\|_{(e)}$,

$$
\text { b) }\left\|\partial_{\alpha} \mathcal{H}_{n}^{a} * f\right\|_{\infty} \leq C\|a\|_{d+1} \times 2^{n|\alpha|} \beta_{e}\left(2^{n d}\right)\|f\|_{\left(e^{*}\right)}
$$

ii) Let $m \in \mathbb{N}_{*}$. There exists a universal constant $C$ (depending on $\alpha, m, d$ and $e$ ) such that

$$
\left\|\mathcal{H}_{n}^{a} * \partial_{\alpha} f\right\|_{(e)} \leq \frac{C\|a\|_{d+1}^{2}}{4^{n m}}\|f\|_{2 m+|\alpha|, 2 m,(e)}
$$

iii) Let $k \in \mathbb{N}$. There exists a universal constant $C$ (depending on $\alpha, k, d$ and e) such that

$$
\left\|\mathcal{H}_{n}^{a} * \partial_{\alpha}(f-g)\right\|_{(e)} \leq C\|a\|_{d+1} \times 2^{n(|\alpha|+k)} \beta\left(2^{n d}\right) d_{k}\left(\mu_{f}, \mu_{g}\right)
$$

iv) For $f, g \in W^{k, e_{*}}$ we define

$$
d_{k, e_{*}}\left(\mu_{f}, \mu_{g}\right)=\sup \left\{\left|\int \phi d \mu_{f}-\int \phi d \mu_{g}\right|: \phi \in W^{k, e_{*}},\|\phi\|_{\left(e_{*}\right)} \leq 1\right\} .
$$

Let $k \in \mathbb{N}$. There exists a universal constant $C$ (depending on $\alpha, k, d$ and $e$ ) such that

$$
\left\|\mathcal{H}_{n}^{a} * \partial_{\alpha}(f-g)\right\|_{(e)} \leq C\|a\|_{d+1} \times 2^{n(|\alpha|+k)} d_{k, e_{*}}\left(\mu_{f}, \mu_{g}\right) .
$$


Remark 2.13 Let us try to explain the gain with respect to the standard projection on the basis of Hermite functions. We have to compare $\mathcal{H}_{n}^{a}$ with $\mathcal{H}_{4^{n}}$ because $\mathcal{H}_{n}^{a}$ is a mixture of projections between $4^{n-1}$ and $4^{n+1}$. Suppose that we work in $L^{p}$ so $e_{p}(x)=|x|^{p}$. In this case $\beta_{e}(t)=C \times t^{1 / p_{*}}$ (with $p_{*}$ the conjugate of $p$ ) so the inequality (2.22), with $k=1$, reads

$$
\left\|\mathcal{H}_{n}^{a} * \partial_{\alpha}(f-g)\right\|_{p} \leq C 2^{n\left(|\alpha|+1+\frac{d}{p_{*}}\right)} d_{1}\left(\mu_{f}, \mu_{g}\right)
$$

In some notes (which are not reported here) we were able to prove that

$$
\left\|\mathcal{H}_{4^{n}} * \partial_{\alpha}(f-g)\right\|_{2} \leq C 2^{n\left(|\alpha|+1+\frac{d}{2}\right)} d_{1}\left(\mu_{f}, \mu_{g}\right)
$$

If we take $p=2$ in 2.24) we obtain exactly (2.25) so there is no gain. But we could not obtain the estimates in (2.25) for any $p$, but only for $p=2$. Here we are able to take any $p>1$ so $p_{*}$ may be taken arbitrary large - and this destroys the dimension $d$.

Proof. i) Using (2.15)

$$
\left|\partial_{\alpha} \mathcal{H}_{n}^{a} * f(x)\right| \leq C 2^{n(|\alpha|+d)}\|a\|_{d+1} \int \rho_{n, d+1}(x-y)|f(y)| d y .
$$

Since $e$ is symmetric $e(|x|)=e(x)$ so that $\|f\|_{(e)}=\||f|\|_{(e)}$. Moreover, if $0 \leq f(x) \leq g(x)$ then $\|f\|_{(e)} \leq\|g\|_{(e)}$. Using these properties and (2.26) and (2.17) we obtain

$$
\begin{aligned}
\left\|\partial_{\alpha} \mathcal{H}_{n}^{a} * f\right\|_{(e)} & =\left\|\partial_{\alpha} \mathcal{H}_{n}^{a} * f\left|\left\|_{(e)} \leq C 2^{n(|\alpha|+d)}\right\| a\left\|_{d+1}\right\| \rho_{n, d+1} *\right| f \mid\right\|_{(e)} \\
& \leq C 2^{n(|\alpha|+d)}\|a\|_{d+1}\left\|\rho_{n, d+1}\right\|_{1}\||f|\|_{(e)} .
\end{aligned}
$$

Using (2.19) with $e(x)=|x|$ we obtain $\left\|\rho_{n, d+1}\right\|_{1} \leq C / 2^{\text {nd }}$. So we conclude that

$$
\left\|\partial_{\alpha} \mathcal{H}_{n}^{a} * f\right\|_{(e)} \leq C\|a\|_{d+1} 2^{n|\alpha|}\|f\|_{(e)}
$$

so a) is proved. Again by (2.26)

$$
\begin{aligned}
\left|\partial_{\alpha} \mathcal{H}_{n}^{a} * f(x)\right| & \leq C\|a\|_{d+1} 2^{n(|\alpha|+d)} \int \rho_{n, d+1}(x-y)|f(y)| d y \\
& \leq C\|a\|_{d+1} 2^{n(|\alpha|+d)}\left\|\rho_{n, d+1}\right\|_{(e)}\|f\|_{\left(e_{*}\right)},
\end{aligned}
$$

the second inequality being a consequence of the Hölder inequality. Using (2.19), b) is proved as well.

ii) We define the functions $a_{m}(t)=a(t) t^{-m}$. Since $a(t)=0$ for $t \leq \frac{1}{4}$ and for $t \geq 4$ we have $\left\|a_{m}\right\|_{d+1} \leq C_{m, d}\|a\|_{d+1}$. Moreover $D \mathcal{H}_{j} * v=(2 j+d) \mathcal{H}_{j} * v$ so we obtain

$$
\mathcal{H}_{j} * v=\frac{1}{2 j}(D-d) \mathcal{H}_{j} * v
$$

We denote $L_{m, \alpha}=(D-d)^{m} \partial_{\alpha}$ and we notice that $L_{m, \alpha}=\sum_{|\beta| \leq 2 m} \sum_{|\gamma| \leq 2 m+|\alpha|} c_{\beta, \gamma} x^{\beta} \partial_{\gamma}$ where $c_{\beta, \gamma}$ are universal constants. It follows that there exists some universal constant $C$ such that

$$
\left\|L_{m, \alpha} f\right\|_{(e)} \leq C\|f\|_{2 m+|\alpha|, 2 m,(e)}
$$


We take now $v \in L^{e_{*}}$ and we write

$$
\begin{aligned}
\left\langle v, \mathcal{H}_{n}^{a} *\left(\partial_{\alpha} f\right)\right\rangle & =\left\langle\mathcal{H}_{n}^{a} * v, \partial_{\alpha} f\right\rangle=\sum_{j=0}^{\infty} a\left(\frac{j}{4^{n}}\right)\left\langle\mathcal{H}_{j} * v, \partial_{\alpha} f\right\rangle \\
& =\sum_{j=1}^{\infty} a\left(\frac{j}{4^{n}}\right) \frac{1}{(2 j)^{m}}\left\langle(D-d)^{m} \mathcal{H}_{j} * v, \partial_{\alpha} f\right\rangle \\
& =\frac{1}{2^{m}} \times \frac{1}{4^{n m}} \sum_{j=1}^{\infty} a_{m}\left(\frac{j}{4^{n}}\right)\left\langle\mathcal{H}_{j} * v, L_{m, \alpha} f\right\rangle \\
& =\frac{1}{2^{m}} \times \frac{1}{4^{n m}}\left\langle\mathcal{H}_{n}^{a_{m}} * v, L_{m, \alpha} f\right\rangle .
\end{aligned}
$$

Using the decomposition form Proposition 2.9 we write $L_{m, \alpha} f=\sum_{j=0}^{\infty} \mathcal{H}_{j}^{a} * L_{m, \alpha} f$. For $j<n-1$ and for $j>n+1$ we have $\left\langle\mathcal{H}_{n}^{a_{m}} * v, \mathcal{H}_{j}^{a} * L_{m, \alpha} f\right\rangle=0$. So using Hölder's inequality

$$
\begin{aligned}
\left|\left\langle v, \mathcal{H}_{n}^{a} *\left(\partial_{\alpha} f\right)\right\rangle\right| & \leq \frac{1}{4^{n m}} \sum_{j=n-1}^{n+1}\left|\left\langle\mathcal{H}_{n}^{a_{m}} * v, \mathcal{H}_{j}^{a} * L_{m, \alpha} f\right\rangle\right| \\
& \leq \frac{1}{4^{n m}} \sum_{j=n-1}^{n+1}\left\|\mathcal{H}_{n}^{a_{m}} * v\right\|_{\left(e_{*}\right)}\left\|\mathcal{H}_{j}^{a} * L_{m, \alpha} f\right\|_{(e)} .
\end{aligned}
$$

Using the point i) a) with $\alpha$ the void index we obtain $\left\|\mathcal{H}_{n}^{a_{m}} * v\right\|_{\left(e_{*}\right)} \leq C\left\|a_{m}\right\|_{d+1}\|v\|_{\left(e_{*}\right)} \leq$ $C \times C_{m, d}\|a\|_{d+1}\|v\|_{\left(e_{*}\right)}$. Moreover, we have $\left\|\mathcal{H}_{j}^{a} * L_{m, \alpha} f\right\|_{(e)} \leq C\|a\|_{d+1}\left\|L_{m, \alpha} f\right\|_{(e)} \leq$ $C\|a\|_{d+1}\|f\|_{2 m+|\alpha|, 2 m,(e)}$, the last inequality being a consequence of (2.27). We obtain

$$
\left|\left\langle v, \mathcal{H}_{n}^{a} *\left(\partial_{\alpha} f\right)\right\rangle\right| \leq \frac{C\|a\|_{d+1}^{2}}{4^{n m}}\|v\|_{e_{*}}\|f\|_{2 m+|\alpha|, 2 m,(e)}
$$

and, since $L^{e}$ is reflexive, (2.21) is proved.

iii) We write

$$
\begin{aligned}
\left|\left\langle v, \mathcal{H}_{n}^{a} *\left(\partial_{\alpha}(f-g)\right)\right\rangle\right| & =\left|\left\langle\mathcal{H}_{n}^{a} * v, \partial_{\alpha}(f-g)\right\rangle\right|=\left|\left\langle\partial_{\alpha} \mathcal{H}_{n}^{a} * v, f-g\right)\right\rangle \mid \\
& =\left|\int \partial_{\alpha} \mathcal{H}_{n}^{a} * v d \mu_{f}-\int \partial_{\alpha} \mathcal{H}_{n}^{a} * v d \mu_{g}\right| .
\end{aligned}
$$

We use (2.20) b) and we obtain

$$
\begin{aligned}
& \left|\int \partial_{\alpha} \mathcal{H}_{n}^{a} * v d \mu_{f}-\int \partial_{\alpha} \mathcal{H}_{n}^{a} * v d \mu_{g}\right| \leq\left\|\partial_{\alpha} \mathcal{H}_{n}^{a} * v\right\|_{k, \infty} d_{k}\left(\mu_{f}, \mu_{g}\right) \\
\leq & \left\|\mathcal{H}_{n}^{a} * v\right\|_{k+|\alpha|, \infty} d_{k}\left(\mu_{f}, \mu_{g}\right) \leq C\|a\|_{d+1} 2^{n(k+|\alpha|)} \beta_{e}\left(2^{n d}\right)\|v\|_{\left(e_{*}\right)} d_{k}\left(\mu_{f}, \mu_{g}\right)
\end{aligned}
$$

which implies (2.22). 
iv) We use (2.20) a) and we obtain

$$
\begin{aligned}
\left|\int \partial_{\alpha} \mathcal{H}_{n}^{a} * v d \mu_{f}-\int \partial_{\alpha} \mathcal{H}_{n}^{a} * v d \mu_{g}\right| & \leq\left\|\partial_{\alpha} \mathcal{H}_{n}^{a} * v\right\|_{k,\left(e_{*}\right)} d_{k, e_{*}}\left(\mu_{f}, \mu_{g}\right) \\
& \leq\left\|\mathcal{H}_{n}^{a} * v\right\|_{k+|\alpha|,\left(e_{*}\right)} d_{k, e_{*}}\left(\mu_{f}, \mu_{g}\right) \\
& \leq C\|a\|_{d+1} 2^{n(k+|\alpha|)}\|v\|_{\left(e_{*}\right)} d_{k, e_{*}}\left(\mu_{f}, \mu_{g}\right) .
\end{aligned}
$$

So $\left\|\mathcal{H}_{n}^{a} *\left(\partial_{\alpha}(f-g)\right)\right\|_{(e)} \leq C 2^{n(k+|\alpha|)} d_{k, e_{*}}\left(\mu_{f}, \mu_{g}\right)$ and iv) is proved.

We are now ready to give the "balance". For $\mu \in \mathcal{M}$ and $\mu_{n}(x)=f_{n}(x) d x, n \in \mathbb{N}$ we recall that

$$
\pi_{q, k, m, e}\left(\mu,\left(\mu_{n}\right)_{n}\right)=\sum_{n=0}^{\infty} 2^{n(q+k)} \beta_{e}\left(2^{n d}\right) d_{k}\left(\mu, \mu_{n}\right)+\sum_{n=0}^{\infty} \frac{1}{2^{2 n m}}\left\|f_{n}\right\|_{2 m+q, 2 m,(e)} .
$$

We also set

$$
\widetilde{\pi}_{q, k, m, e}\left(\mu,\left(\mu_{n}\right)_{n}\right)=\sum_{n=0}^{\infty} 2^{n(q+k)} d_{k, e}\left(\mu, \mu_{n}\right)+\sum_{n=0}^{\infty} \frac{1}{2^{2 n m}}\left\|f_{n}\right\|_{2 m+q, 2 m,(e)} .
$$

Proposition 2.14 Let $q, k \in \mathbb{N}, m \in \mathbb{N}_{*}$ and $e \in \mathcal{E}$. There exists a universal constant $C$ (depending on $q, k, m, d$ and $e$ ) such that for every $f, f_{n} \in C^{2 m+q}\left(\mathbb{R}^{d}\right), n \in \mathbb{N}$

$$
\begin{aligned}
\text { a) }\|f\|_{q,(e)} & \leq C \pi_{q, k, m, e}\left(\mu,\left(\mu_{n}\right)_{n}\right), \\
\text { b) }\|f\|_{q,(e)} & \leq C \widetilde{\pi}_{q, k, m, e}\left(\mu,\left(\mu_{n}\right)_{n}\right) .
\end{aligned}
$$

Here $\mu(x)=f(x) d x$ and $\mu_{n}(x)=f_{n}(x) d x$.

Proof Let $\alpha$ with $|\alpha| \leq q$. Using Proposition 2.9

$$
\partial_{\alpha} f=\sum_{n=1}^{\infty} \mathcal{H}_{n}^{a} * \partial_{\alpha} f=\sum_{n=1}^{\infty} \mathcal{H}_{n}^{a} * \partial_{\alpha}\left(f-f_{n}\right)+\sum_{n=1}^{\infty} \mathcal{H}_{n}^{a} * \partial_{\alpha} f_{n}
$$

and using (2.22) and (2.21)

$$
\begin{aligned}
\left\|\partial_{\alpha} f\right\|_{(e)} & \leq \sum_{n=1}^{\infty}\left\|\mathcal{H}_{n}^{a} * \partial_{\alpha}\left(f-f_{n}\right)\right\|_{(e)}+\sum_{n=1}^{\infty}\left\|\mathcal{H}_{n}^{a} * \partial_{\alpha} f_{n}\right\|_{(e)} \\
& \leq C \sum_{n=1}^{\infty} 2^{n(|\alpha|+k)} \beta_{e}\left(2^{n d}\right) d_{k}\left(\mu_{f}, \mu_{f_{n}}\right)+C \sum_{n=1}^{\infty} \frac{1}{2^{2 n m}}\left\|f_{n}\right\|_{2 m+|\alpha|, 2 m,(e)}
\end{aligned}
$$

so (2.28) a) is proved. The same reasoning, using (2.23) gives (2.28) b).

We can now give the

Proof of Theorem 2.3. Step 1. Regularization. For $\delta \in(0,1)$ we consider $\gamma_{\delta}$ the density of the centred Gaussian probability measure with variance $\delta$. Moreover we 
consider a truncation function $\Phi_{\delta} \in C^{\infty}$ such that $1_{B_{\delta^{-1}}(0)} \leq \Phi_{\delta} \leq 1_{B_{1+\delta^{-1}}(0)}$ and whose derivatives of all orders are bounded uniformly w.r.t. $\delta$. Then we define

$$
\begin{aligned}
& T_{\delta}: C^{\infty} \rightarrow C^{\infty}, \quad T_{\delta} f=\left(\Phi_{\delta} f\right) * \gamma_{\delta} \\
& \widetilde{T}_{\delta}: C^{\infty} \rightarrow C_{c}^{\infty}, \quad \widetilde{T}_{\delta} f=\Phi_{\delta}\left(f * \gamma_{\delta}\right) \text {. }
\end{aligned}
$$

Moreover, for a measure $\mu \in \mathcal{M}$ we define $T_{\delta}^{*} \mu$ by

$$
\left\langle T_{\delta}^{*} \mu, \phi\right\rangle=\left\langle\mu, T_{\delta} \phi\right\rangle \text {. }
$$

Then $T_{\delta}^{*} \mu$ is an absolute continuous measure with density $p_{T_{\delta}^{*} \mu} \in C_{c}^{\infty}$ given by

$$
p_{T_{\delta}^{*} \mu}(y)=\Phi_{\delta}(y) \int \gamma_{\delta}(x-y) d \mu(x)
$$

Step 2. We prove that for every $\mu \in \mathcal{M}$ and $\mu_{n}(d x)=f_{n}(x) d x, n \in \mathbb{N}$ we have

$$
\pi_{q, k, m, e}\left(T_{\delta}^{*} \mu,\left(T_{\delta}^{*} \mu_{n}\right)_{n}\right) \leq C \pi_{q, k, m, e}\left(\mu,\left(\mu_{n}\right)_{n}\right) .
$$

Since $\left\|T_{\delta} \phi\right\|_{k, \infty} \leq C\|\phi\|_{k, \infty}$ one has $d_{k}\left(T_{\delta}^{*} \mu, T_{\delta}^{*} \mu_{n}\right) \leq C d_{k}\left(\mu, \mu_{n}\right)$.

For $\mu_{n}(d x)=f_{n}(x) d x$ we have $p_{T_{\delta}^{*} \mu_{n}}(y)=\widetilde{T}_{\delta} f_{n}$ Let us now check that

$$
\left\|\widetilde{T}_{\delta} f_{n}\right\|_{2 m+q, 2 m,(e)} \leq C\left\|f_{n}\right\|_{2 m+q, 2 m,(e)} .
$$

For a measurable function $g: \mathbb{R}^{d} \rightarrow \mathbb{R}$ and for $\lambda \geq 0$ we denote $g_{\lambda}(x)=(1+|x|)^{\lambda} g(x)$. Since $(1+|x|)^{\lambda} \leq(1+|y|)^{\lambda}(1+|x-y|)^{\lambda}$ it follows that

$$
\left(\widetilde{T}_{\delta} g\right)_{\lambda}(x)=(1+|x|)^{\lambda} \Phi_{\delta}(x) \int_{\mathbb{R}^{d}} \gamma_{\delta}(y) g(x-y) d y \leq \int_{\mathbb{R}^{d}} \gamma_{\delta, \lambda}(y) g_{\lambda}(x-y) d y=\gamma_{\delta, \lambda} * g_{\lambda}(x)
$$

Then by (2.17) $\left\|\left(T_{\delta} g\right)_{\lambda}\right\|_{e} \leq\left\|\gamma_{\delta, \lambda} * g_{\lambda}\right\|_{e} \leq C\left\|\gamma_{\delta, \lambda}\right\|_{1}\left\|g_{\lambda}\right\|_{e} \leq C\left\|g_{\lambda}\right\|_{e}$. Using this inequality (with $\lambda=2 m$ ) for $g=\partial_{\alpha} f_{n}$ we obtain (2.31). And (2.30) follows.

Step 3. Let $\mu \in \mathcal{S}_{q, k, m, e}$ so that $\rho_{q, k, m, e}(\mu)<\infty$. Using (2.28), a) we have $\left\|T_{\delta}^{*} \mu\right\|_{W^{q,(e)}} \leq$ $\rho_{q, k, m, e}\left(T_{\delta}^{*} \mu\right)$ and moreover, using and (2.30)

$$
\sup _{\delta \in(0,1)}\left\|T_{\delta}^{*} \mu\right\|_{W^{q,(e)}} \leq C \sup _{\delta \in(0,1)} \rho_{q, k, m, e}\left(T_{\delta}^{*} \mu\right) \leq \rho_{q, k, m, e}(\mu)<\infty .
$$

So the family $T_{\delta}^{*} \mu, \delta \in(0,1)$ is bounded in $W^{q, e}$ which is a reflexive space. So it is weakly relative compact. Consequently we may find a sequence $\delta_{n} \rightarrow 0$ such that $T_{\delta_{n}}^{*} \mu \rightarrow f \in$ $W^{q, e}$ weakly. It is easy to check that $T_{\delta_{n}}^{*} \mu \rightarrow \mu$ weakly so $\mu(d x)=f(x) d x$ and $f \in W^{q, e}$. As a consequence of (2.32) we have $\|\mu\|_{W^{q,(e)}} \leq C \rho_{q, k, m, e}(\mu)$.

\subsection{Integration by parts formulas}

In order to use the criterion presented in the previous section one needs to have estimates for the densities of the approximating measures $\mu_{n}$. Sometimes these densities are explicit 
and then there is no problem. But sometimes they are not and then the integration by parts machinery is very useful - we present it in this section. An example related to diffusion processes is given in Section 4, if an ellipticity condition holds then one may exhibit a Gaussian random variable but under Hörmander condition this is no more possible.

We recall that $\mathcal{M}_{+}$is the set of positive and finite measures on $\mathbb{R}^{d}$ with the Borel $\sigma$-field and $L_{\mu}^{p}:=L^{p}\left(\mathbb{R}^{d}, d \mu\right)$. For $\mu \in \mathcal{M}_{+}, m \in \mathbb{N}, p \geq 1$, we define the Sobolev space $W_{\mu}^{m, p}$ to be the space of the measurable functions $g: \mathbb{R}^{d} \rightarrow \mathbb{R}$ such that for every multi index $\alpha$ with $|\alpha| \leq m$ there exists a function $\theta_{\alpha}(g) \in L_{\mu}^{p}$ such that

$$
\int \partial_{\alpha} f \times g d \mu=(-1)^{|\alpha|} \int f \times \theta_{\alpha}(g) d \mu \quad \forall f \in C_{c}^{\infty}\left(\mathbb{R}^{d}\right) .
$$

We denote

$$
\partial_{\alpha}^{\mu} g=\theta_{\alpha}(g)
$$

Notice that $\partial_{\alpha}^{\mu}$ is not a differential operator. Indeed it is easy to check that this operator verifies the following computation rules (see Lemma 9 in [1]). Let $\phi \in W_{\mu}^{1, p}$ and $\psi \in$ $C_{b}^{1}\left(\mathbb{R}^{d}\right)$. Then $\phi \psi \in W_{\mu}^{1, p}$ and

$$
\partial_{i}^{\mu}(\phi \psi)=\psi \partial_{i}^{\mu} \phi+\phi \partial_{i} \psi
$$

Taking $\phi=1$ we obtain

$$
\partial_{i}^{\mu} \psi=\psi \partial_{i}^{\mu} 1+\partial_{i} \psi
$$

We define the Sobolev norm

$$
\|g\|_{W_{\mu}^{m, p}}=\|g\|_{L_{\mu}^{p}}+\sum_{1 \leq|\alpha| \leq m}\left\|\partial_{\alpha}^{\mu} g\right\|_{L_{\mu}^{p}}
$$

and for $p>d$ we denote

$$
c_{m, p}(\mu)=\|1\|_{W_{\mu}^{1, p}}^{k_{d, p}}\|1\|_{W_{\mu}^{m, p}} \quad \text { with } \quad k_{d, p}=\frac{d-1}{1-d / p} .
$$

The corresponding definition in terms of random variables is given by means of the usual integration by parts formulas. On a probability space $(\Omega, \mathcal{F}, P)$ we consider a $d$ dimensional $p$ integrable random variable $F$ and a one dimensional $p$ integrable random variable $G$. Suppose that for each $\alpha$ with $|\alpha| \leq m$ there exists a $p$ integrable random variable $H_{\alpha}(F, G)$ such that

$$
\left.\mathbb{E}\left(\partial_{\alpha} f(F) G\right)\right)=(-1)^{|\alpha|} \mathbb{E}\left(f(F) H_{\alpha}(F, G)\right) \quad \forall f \in C_{c}^{\infty}\left(\mathbb{R}^{d}\right) .
$$

If $\mu_{F}$ is the law of $F$ and $g(x)=\mathbb{E}(G \mid F=x)$ then the above integration by parts formula is equivalent to $g \in W_{\mu_{F}}^{m, p}$ and $\theta_{\alpha}(g)=\mathbb{E}\left(H_{\alpha}(F, G) \mid F=x\right)$. Indeed, taking conditional expectations

$$
\begin{aligned}
\int\left(\partial_{\alpha} f \times g\right) d \mu_{F} & \left.=\mathbb{E}\left(\partial_{\alpha} f(F) \mathbb{E}(G \mid F)\right)=\mathbb{E}\left(\partial_{\alpha} f(F) G\right)\right) \\
& =(-1)^{|\alpha|} \mathbb{E}\left(f(F) H_{\alpha}(F, G)\right)=(-1)^{|\alpha|} \mathbb{E}\left(f(F) \mathbb{E}\left(H_{\alpha}(F, G) \mid F\right)\right) \\
& =\int\left(f \times \theta_{\alpha}(g)\right) d \mu_{F} .
\end{aligned}
$$


Moreover, since $\left|\mathbb{E}\left(H_{\alpha}(F, G) \mid F=x\right)\right|^{p} \leq \mathbb{E}\left(\left|H_{\alpha}(F, G)\right|^{p} \mid F=x\right)$ we have

$$
\|g\|_{W_{\mu}^{m, p}}^{p} \leq \mathbb{E}|G|^{p}+\sum_{1 \leq|\alpha| \leq m} \mathbb{E}\left|H_{\alpha}(F, G)\right|^{p}
$$

We will express our results in terms of positive measures but, in view of the above inequality, everything translates immediately in terms of random variables.

Remark 2.15 An analogous formalism has been introduced by Sigekawa [25] and by Malliavin and Thalmaier [19] ( $\partial_{\alpha}^{\mu} g$ corresponds to the so called "covering vector fields" from [19]). Here we follow [1].

We introduce now the Poisson kernel on $\mathbb{R}^{d}$. It is given by

$$
Q_{2}(x)=a_{2} \ln |x|, \quad Q_{d}(x)=\frac{a_{d}}{|x|^{d-2}}, d>2
$$

where $a_{i}$ are some normalization constants. In order to include the one dimensional case we denote $Q_{1}(x)=x_{+}$. The kernel $Q_{d}$ is the fundamental solution to the equation $\triangle Q_{d}=\delta_{0}$. In [1] Theorem 5 we prove the following estimate. Suppose that $1 \in W_{\mu}^{1, p}$ for some $p>d$. Then

$$
\Theta_{p}(\mu):=\sup _{x \in \mathbb{R}^{d}} \sum_{i=1}^{d}\left(\mathbb{E} \int_{\mathbb{R}^{d}}\left|\partial_{i} Q_{d}(x-y)\right|^{\frac{p}{p-1}} d \mu(x)\right)^{(p-1) / p} \leq C\|1\|_{W_{\mu}^{1, p}}^{k_{d, p}} .
$$

with $k_{d, p}$ given in (2.36) and $C$ a universal constant.

Following the idea of Malliavin and Thalmaier [19], in [1] we used the kernel $Q_{d}$ in order to give a representation theorem for the density of $\mu$. Consider a function $\psi \in C_{b}^{\infty}\left(\mathbb{R}^{d}\right)$ such that $1_{B_{1}(0)} \leq \psi \leq 1_{B_{2}(0)}$ with $B_{r}(0)=\{x:|x|<r\}$. For a fixed $x \in \mathbb{R}^{d}$ we denote $\psi_{x}(y)=\psi(x-y)$. Proposition 9 from [1] says that, if $1 \in W_{\mu}^{1, p}$ for some $p>d$, then $\mu(d x)=p_{\mu}(x) d x$ with

$$
p_{\mu}(x)=\sum_{i=1}^{d} \int \partial_{i} Q_{d}(y-x) \partial_{i}^{\mu} \psi_{x}(y) 1_{\{|x-y|<2\}} \mu(d y)
$$

Notice that by (2.35), if $1 \in W_{\mu}^{1, p}$ then $\psi_{x} \in W_{\mu}^{1, p}$ and $\partial_{i}^{\mu} \psi_{x}$ has the support included in $B_{2}(x)$. So $1_{\{|x-y|<2\}}$ appears just to emphasize this fact.

Suppose now that $1 \in W_{\mu}^{m, p}$ for some $m \in \mathbb{N}$ and $p>d$. In Theorem 8 from [1] we prove that $p_{\mu} \in C^{m-1}\left(\mathbb{R}^{d}\right)$. And it is easy to see that for a multi index $\alpha$ with $|\alpha| \leq m-1$ one has

$$
\partial_{\alpha} p_{\mu}(x)=(-1)^{|\alpha|} \sum_{i=1}^{d} \int \partial_{i} Q_{d}(y-x) \partial_{\alpha}^{\mu} \partial_{i}^{\mu} \psi_{x}(y) 1_{\{|x-y|<2\}} \mu(d y) .
$$

We need the following variant of Proposition 9 in [1]. 
Proposition 2.16 Suppose that $1 \in W_{\mu}^{m, p}$ for some $m \in \mathbb{N}_{*}$ and $p>d$. Then $\mu(d x)=$ $p_{\mu}(x) d x$ with $p_{\mu} \in C^{m-1}\left(\mathbb{R}^{d}\right)$ and for every multi index $\alpha$ with $|\alpha| \leq m-1$ and $k \in \mathbb{N}$

$$
\left|\partial_{\alpha} p_{\mu}(x)\right| \leq C \times c_{m, 2 p}(\mu) m_{k}^{\frac{1}{2}}(\mu) u_{k / 2}(x)
$$

with $u_{k}(x)=(1+|x|)^{-k}$ and

$$
m_{k}(\mu)=\int(1+|x|)^{k} d \mu(x)
$$

Proof. We write

$$
\left|\partial_{\alpha} p_{\mu}(x)\right| \leq \sum_{i=1}^{d} I_{i} \times J_{i} \leq\left(\sum_{i=1}^{d} I_{i}^{2}\right)^{1 / 2}\left(\sum_{i=1}^{d} J_{i}^{2}\right)^{1 / 2}
$$

with

$$
I_{i}=\left(\int\left|\partial_{i} Q_{d}(y-x)\right|^{\frac{p}{p-1}} \mu(d y)\right)^{\frac{p-1}{p}} \quad \text { and } \quad J_{i}=\left(\int\left|\partial_{\alpha}^{\mu} \partial_{i}^{\mu} \psi_{x}(y)\right|^{p} 1_{\{|x-y|<2\}} \mu(d y)\right)^{\frac{1}{p}} .
$$

We use (2.39) and we obtain

$$
\left(\sum_{i=1}^{d} I_{i}^{2}\right)^{1 / 2} \leq C\|1\|_{W_{\mu}^{1, p}}^{k_{d, p}}
$$

Moreover using Schwarz inequality

$$
J_{i} \leq\left(\int\left|\partial_{\alpha}^{\mu} \partial_{i}^{\mu} \psi_{x}(y)\right|^{2 p} \mu(d y)\right)^{\frac{1}{2 p}} \mu\left(B_{2}(x)\right)^{\frac{1}{2}} .
$$

Using repeatedly (2.35) one can check that $\left\|\psi_{x}\right\|_{W_{\mu}^{m, 2 p}} \leq C_{\psi}\|1\|_{W_{\mu}^{m, 2 p}}$ where $C_{\psi}$ is a universal constant which depends on the derivatives of $\psi$ up to order $m$. Take now a random variable $\xi$ of law $\mu$. We may assume without loss of generality that $|x| \geq 4$ and then, using Chebyshev inequality

$$
\mu\left(B_{2}(x)\right)=\mathbb{P}\left(\xi \in B_{2}(x)\right) \leq \mathbb{P}\left(|\xi| \geq \frac{|x|}{2}\right) \leq \frac{C}{|x|^{k}} m_{k}(\mu) .
$$

So $J_{i} \leq C\|\mu\|_{m, 2 p} m_{k}^{1 / 2}(\mu) u_{k / 2}(x)$. We conclude that

$$
\left|\partial_{\alpha} p_{\mu}(x)\right| \leq C\|1\|_{W_{\mu}^{1, p}}^{k_{d, p}}\|1\|_{W_{\mu}^{m, 2 p}} m_{k}^{1 / 2}(\mu) u_{k / 2}(x)=c_{m, 2 p}(\mu) m_{k}^{1 / 2}(\mu) u_{k / 2}(x)
$$

and consequently (2.41) is proved.

By using Remark 2.1, as an immediate consequence of (2.41) (with $p=d+1$ ) we obtain

$$
\left\|p_{\mu}\right\|_{2 m+q, 2 m,(e)} \leq C_{d, e} c_{2 m+q, 2(d+1)}(\mu) m_{2(d+1+m)}^{1 / 2}(\mu) .
$$

We can now re-formulate the results from Section 2.1. We define

$$
\widetilde{M}_{m, q, e}(R)=\left\{\mu \in \mathcal{M}_{a}\left(\mathbb{R}^{d}\right): c_{2 m+q, 2(d+1)}(\mu) m_{2(d+1+m)}^{1 / 2}(\mu) \leq R\right\}
$$


and by (2.41) we obtain $\widetilde{M}_{m, q, e}(R) \subset M_{m, q, e}(C \times R)$ where $C$ is a universal constant. Then we consider the following

Hypothesis $\widetilde{H}_{q}(k, m, e)$. For $q, k \in \mathbb{N}$ and $m \in \mathbb{N}_{*}$ there exists a $>1$ such that

$$
\varlimsup_{R \rightarrow \infty} \frac{L_{a}(R)^{1+\frac{k+q}{2 m}} \beta_{e}\left(L_{a}(R)^{\frac{d}{2 m}}\right)}{R} d_{k}\left(\mu, \widetilde{M}_{m, q, e}(R)\right)<\infty .
$$

We define

$$
\widetilde{B}_{q}(k, m, e)=\left\{\mu \in \mathcal{M}\left(\mathbb{R}^{d}\right): \widetilde{H}_{q}(k, m, e) \text { holds for } \mu\right\} .
$$

Our criterion can be stated as follows.

Theorem 2.17 Let $q, k \in \mathbb{N}, m \in \mathbb{N}_{*}$ and $e \in \mathcal{E}$. Then $\widetilde{B}_{q}(k, m, e) \subset B_{q}(k, m, e) \subset$ $\mathcal{S}_{q, k, m, e} \subset W^{q, e}$.

\section{Interpolation spaces}

We give a variant of the results from the previous section in terms of interpolation spaces. More precisely we will prove that the space $\mathcal{S}_{q, k, m, e}$ considered in Section 2.1 (2.10) is an interpolation space.

To begin we recall the framework of interpolation spaces. We are given two Banach spaces $\left(X,\|\circ\|_{X}\right),\left(Y,\|\circ\|_{Y}\right)$ with $X \subset Y$ (with continuous embedding). We denote $\mathcal{L}(X, X)$ the space of the linear bounded operators from $X$ into itself and we denote by $\|L\|_{X, X}$ the operator norm. A Banach space $\left(W,\|\circ\|_{W}\right)$ such that $X \subset W \subset Y$ is called an interpolation space for $X$ and $Y$ if $\mathcal{L}(X, X) \cap \mathcal{L}(Y, Y) \subset \mathcal{L}(W, W)$. Let $\gamma \in(0,1)$. If there exists a constant $C$ such that $\|L\|_{W, W} \leq C\|L\|_{X, X}^{\gamma}\|L\|_{Y, Y}^{1-\gamma}$ for every $L \in \mathcal{L}(X, X) \cap \mathcal{L}(Y, Y)$ then $W$ is an interpolation space of order $\gamma$. And if one may take $C=1$ then $W$ is an exact interpolation space of order $\gamma$. There are several methods for constructing interpolation spaces. We focus here on the so called $K$-method. For $y \in Y$ and $t>0$ one defines $K(y, t)=\inf _{x \in X}\left(\|y-x\|_{Y}+t\|x\|_{X}\right)$ and

$$
\|y\|_{\gamma}=\int_{0}^{\infty} t^{-\gamma} K(y, t) \frac{d t}{t}, \quad(X, Y)_{\gamma}=\left\{y \in Y:\|y\|_{\gamma}<\infty\right\}
$$

Then one proves that $(X, Y)_{\gamma}$ is an exact interpolation space of order $\gamma$.

In the theory of interpolation spaces one considers a more general situation: one does not require that $X \subset Y$ but instead one works with $X \cap Y$ and $X+Y$. Moreover one considers a whole family of norms given by $\|y\|_{\gamma, q}^{q}=\int_{0}^{\infty}\left(t^{-\gamma} K(y, t)\right)^{q} \frac{d t}{t}$ with $\infty>q \geq 1$. In our framework we are concerned with $X \subset Y$ and $q=1$ so we leave out the general setting. But we will need a variant of the norm $\|y\|_{\gamma}$ : for $\gamma \in(0,1)$ and $b \geq 0$ we define

$$
\|y\|_{\gamma, b}=\int_{0}^{\infty} t^{-\gamma}|\ln t|^{b} K(y, t) \frac{d t}{t} \quad \text { and } \quad|y|_{\gamma, b}=\int_{0}^{1} t^{-\gamma}|\ln t|^{b} K(y, t) \frac{d t}{t} .
$$

Notice that $K(y, t) \leq\|y\|_{Y}$ so if $\gamma>0$ then

$$
|y|_{\gamma, b} \leq\|y\|_{\gamma, b} \leq|y|_{\gamma, b}+C_{\gamma, b}\|y\|_{Y} .
$$


Therefore we may work with one or another of these two norms. But if $\gamma=0$ then $\int_{1}^{\infty}|\ln t|^{b} K(t, y) \frac{d t}{t}$ may be infinite. This is why we prefer to work with $|y|_{\gamma, b}$. We define

$$
K_{\gamma, b}(X, Y)=\left\{y \in Y:|y|_{\gamma, b}<\infty\right\}
$$

In the case $\gamma>0$ we have $K_{\gamma, b}(X, Y)=\left\{y \in Y:\|y\|_{\gamma, b}<\infty\right\}$ and moreover if $b=0$ we have $\|y\|_{\gamma}=\|y\|_{\gamma, 0}$ and $(X, Y)_{\gamma}=K_{\gamma, 0}(X, Y)$.

We introduce now another space which is the analogous of the space $\mathcal{S}_{q, k, m, e}$ defined in (2.10). Let $a, \theta \geq 0$ and $m \in \mathbb{N}_{*}$. For $y \in Y$ and for a sequence $x_{n} \in X, n \in \mathbb{N}$ we define

$$
\pi_{\theta, m, a}\left(y,\left(x_{n}\right)_{n}\right)=\sum_{n=1}^{\infty} 2^{n \theta} n^{a}\left\|y-x_{n}\right\|_{Y}+\frac{1}{2^{2 n m}}\left\|x_{n}\right\|_{X} .
$$

Moreover we define

$$
\rho_{\theta, m, a}^{X, Y}(y)=\inf \pi_{\theta, m, a}\left(y,\left(x_{n}\right)_{n}\right)
$$

with the infimum taken over all the sequences $x_{n} \in X, n \in \mathbb{N}$. We define

$$
S_{\theta, m, a}(X, Y)=\left\{y \in Y: \rho_{\theta, m, a}^{X, Y}(y)<\infty\right\} .
$$

It is easy to check that $\rho_{\theta, m, a}^{X, Y}$ is a norm on $S_{\theta, m, a}(X, Y)$.

Finally we define the space which corresponds to the balance: for $\alpha, \beta \geq 0$

$$
B_{\alpha, \beta}(X, Y)=\left\{y \in Y: \varlimsup_{\lim _{R \rightarrow \infty}} R^{\alpha}(\ln R)^{\beta} d_{Y}\left(y, B_{X}(R)\right)<\infty\right\} .
$$

In the appendix we prove the following relation between these spaces (see Proposition 6.4 and Proposition 6.5):

Proposition 3.1 Given $\theta, a \geq 0$ and $m \in \mathbb{N}_{*}$, one has

$$
B_{\alpha, \beta}(X, Y) \subset S_{\theta, m, a}(X, Y)=K_{\gamma, b}(X, Y)
$$

with

$$
\gamma=\frac{\theta}{2 m+\theta}, b=\frac{2 m a}{2 m+\theta} \quad \text { and } \quad \alpha=\frac{\theta}{2 m}, \beta=2+a+\frac{\theta}{m} .
$$

In particular, taking $a=0$ we obtain

$$
B_{\alpha, \beta}(X, Y) \subset(X, Y)_{\gamma} \quad \text { with } \quad \alpha=\frac{\gamma}{1-\gamma}, \beta=\frac{2}{1-\gamma} .
$$

\subsection{Distribution spaces}

We precise now the particular spaces we will work with. We denote by $W^{k, \infty}$ the closure of the $C_{b}^{\infty}$ functions with respect to $\|\circ\|_{k, \infty}$ and we consider the dual space $W_{*}^{k, \infty}$. So $W_{*}^{k, \infty}$ is the space of the bounded linear functionals $u: W^{k, \infty} \rightarrow \mathbb{R}$ with the norm

$$
\|u\|_{W_{*}^{k, \infty}}=\sup \left\{|\langle u, f\rangle|: f \in W^{k, \infty},\|f\|_{k, \infty} \leq 1\right\}
$$


Let $k, q, m \in \mathbb{N}, m>\frac{d}{2}$ and $e \in \mathcal{E}_{\alpha, \gamma}$ for some $\alpha, \gamma \geq 0$ (see (2.11) $)$. We will work with the spaces

$$
X=W^{2 m+q, 2 m, e} \quad \text { and } \quad Y=W_{*}^{k, \infty} .
$$

It is easy to check that, if $l>d$, then $W^{0, l, e} \subset L^{1}$. Indeed if $u_{l}(x)=(1+|x|)^{-l}$ and $v(x)=(1+|x|)^{l} f(x)$ then using Hölder inequality $\int|f(x)| d x=\int\left|u_{l}(x) v(x)\right| d x \leq$ $2\|v\|_{(e)}\left\|u_{l}\right\|_{\left(e_{*}\right)} \leq 2\|f\|_{0, l,(e)}\left\|u_{l}\right\|_{\left(e_{*}\right)}$ and by Remark 2.1 this is finite. So we may embed $X=W^{2 m+q, 2 m, e}$ in $Y=W_{*}^{k, \infty}$ in the sense that $f \in X \rightarrow \tilde{f} \in Y$ with $\langle\tilde{f}, g\rangle=$ $\int f(x) g(x) d x$. Moreover for a measure $\mu \in \mathcal{M}$ we denote by $\widetilde{\mu}$ the linear functional $\langle\widetilde{\mu}, g\rangle=\int g(x) d \mu(x)$. Since $g \in W^{k, \infty}$ is bounded $\langle\widetilde{\mu}, g\rangle$ is well defined. So one has $\widetilde{\mu} \in W_{*}^{k \infty}$. The distance introduced in (2.7) becomes

$$
\begin{aligned}
d_{k}(\mu, \nu) & =\sup \left\{\left|\int \phi d \mu-\int \phi d \nu\right|: \phi \in C^{k}\left(\mathbb{R}^{d}\right),\|\phi\|_{k, \infty} \leq 1\right\} \\
& =\sup \left\{|\langle\widetilde{\mu}-\widetilde{\nu}, \phi\rangle|: \phi \in C^{k}\left(\mathbb{R}^{d}\right),\|\phi\|_{k, \infty} \leq 1\right\}=\|\widetilde{\mu}-\widetilde{\nu}\|_{W_{*}^{k, \infty}} .
\end{aligned}
$$

Recall $\rho_{q, k, m, e}$ defined in (2.9) and take $\theta=q+k+\alpha d, a=\gamma$. Given $e \in \mathcal{E}_{\alpha, \gamma}$ one may find a universal $C$ such that for every $\mu \in \mathcal{M}$ one has

$$
\frac{1}{C} \rho_{q, k, m, e}(\mu) \leq \rho_{\theta, m, a}^{X, Y}(\widetilde{\mu}) \leq C \rho_{q, k, m, e}(\mu) .
$$

We have the following analogous of Theorem 2.3 :

Proposition 3.2 Let $k, q, m \in \mathbb{N}, m>\frac{d}{2}$ and $e \in \mathcal{E}_{\alpha, \gamma}$. We take $\theta=q+k+\alpha d$ and $X=W^{2 m+q, 2 m, e}, Y=W_{*}^{k, \infty}$. Then,

$$
S_{\theta, m, \gamma}(X, Y) \subset W^{q, e} .
$$

The proof is identical with the proof of Theorem 2.3 (we have not used there the fact that $\mu$ is a measure, but only the fact that it is a distribution) so we skip it.

The main result in this section is the following:

Theorem 3.3 Let $k, q, m \in \mathbb{N}$ and $e \in \mathcal{E}_{\alpha, \gamma}$ for some $\alpha, \gamma \geq 0$. We take $\theta=q+k+\alpha d$ and we denote $X=W^{2 m+q, 2 m, e} \subset W_{*}^{k, \infty}=Y$. Then,

$$
\begin{aligned}
& W^{q+1,2 m, e} \subset \mathcal{S}_{q, k, m, e}=S_{\theta, m, \gamma}(X, Y)=K_{\rho, b}(X, Y) \subset W^{q, e} \\
& \text { with } \rho=\frac{\theta}{2 m+\theta}, b=\frac{2 m \gamma}{2 m+\theta},
\end{aligned}
$$

the first inclusion holding for $m>d / 2$, and

$$
B_{u, v}(X, Y) \subset S_{\theta, m, \gamma}(X, Y) \quad u=\frac{\theta}{2 m}, v=2+\gamma+\frac{\theta}{m} .
$$

Proof. As a consequence of the previous proposition the elements of $S_{\theta, m, \gamma}(X, Y)$ are functions. So using (3.5) we obtain $\mathcal{S}_{q, k, m, e}=S_{\theta, m, \gamma}(X, Y)$. The equality $S_{\theta, m, \gamma}(X, Y)=$ $K_{\rho, b}(X, Y)$ and the inclusion $B_{u, v}(X, Y) \subset S_{\theta, m, \gamma}(X, Y)$ are proved in (3.3). The inclusions 
$W^{q+1,2 m, e} \subset S_{\theta, m, \gamma}(X, Y)=K_{\rho, b}(X, Y) \subset W^{q, e}$ follow from Theorem 2.4 and the fact that $S_{\theta, m, \gamma}(X, Y)=\mathcal{S}_{q, k, m, e}$.

We come now back to the examples given in the first section.

Example 1. We take $e_{p}(t)=|t|^{p}$ so that $\beta_{e_{p}}(t)=|t|^{1 / p_{*}}$. It follows that $e_{p} \in \mathcal{E}_{\alpha, \gamma}$ with $\alpha=\frac{1}{p_{*}}$ and $\gamma=0$. The Sobolev spaces associated to $e_{p}$ are the standard Sobolev spaces so we denote $W^{q, p}=W^{q, e_{p}}$ and so on. We also have $b=0$ so $K_{\rho, b}(X, Y)=(X, Y)_{\rho}$ which is the standard interpolation space. Moreover $\theta=q+k+\alpha d=q+k+d / p_{*} \rho=\theta /(2 m+\theta)$ and we obtain

$$
\begin{aligned}
& W^{q+1,2 m, p} \subset \mathcal{S}_{q, k, m, e_{p}}=\left(W^{2 m+q, 2 m, p}, W_{*}^{k, \infty}\right)_{\rho} \subset W^{q, p}, \\
& \text { with } \rho=\frac{q+k+d / p_{*}}{2 m+q+k+d / p_{*}} .
\end{aligned}
$$

Example 2. We take $e_{\log }(t)=(1+|t|) \ln (1+|t|)$. So $e_{\log } \in \mathcal{E}_{0,1}$. Now we work with

$$
K_{\rho, b}(X, Y)=\left\{y \in Y: \int_{0}^{1} \frac{|\ln t|^{b}}{t^{\rho}} K(y, t) \frac{d t}{t}<\infty\right\} .
$$

This is no more a standard interpolation space. The above theorem gives

$$
\begin{aligned}
& W^{q+1,2 m, e_{\log }} \subset \mathcal{S}_{q, k, m, e_{\log }}=K_{\rho, b}\left(W^{2 m+q, 2 m, e_{\log }}, W_{*}^{k, \infty}\right) \subset W^{q, e_{\log }}, \\
& \text { with } \rho=\frac{q+k}{2 m+q+k} \text { and } b=\frac{2 m}{2 m+q+k} .
\end{aligned}
$$

\subsection{Negative Sobolev spaces}

Usually in interpolation theory one discusses about $L^{p}$ spaces and the case of $L^{\infty}$ treated above appears as a limit case which is more delicate. In this section we investigate the results that one may obtain for $L^{p}$ using the machinery based on Hermite expansions (presented in Section 2.2). For simplicity we leave out the Orlicz norms and we restrict ourself to $L^{p}$ spaces. So the spaces we work with are $X=W^{2 m+q, 2 m, p}$ and $Y=W^{-k, p_{*}}=$ $\left(W^{k, p_{*}}\right)^{*}$ the dual of the standard Sobolev space $W^{k, p_{*}}$. The result in this section is the following:

Theorem 3.4 Let $k, q, m \in \mathbb{N}$. We denote $X=W^{2 m+q, 2 m, p} \subset W^{-k, p_{*}}=Y$. Then

$$
W^{q+1,2 m, e} \subset\left(W^{2 m+q, 2 m, p}, W^{-k, p_{*}}\right)_{\rho} \subset W^{q, p} \quad \text { with } \quad \rho=\frac{q+k}{2 m+q+k},
$$

first inclusion holding only for $p_{*}>\frac{d(2 m-1)}{2 m+k+q}$ and $m>d / 2$.

Proof. The proof is the same as for Theorem 2.3 so we sketch it only (we use the notation introduced in that proof).

Let us first prove that $(X, Y)_{\rho} \subset W^{q, p}$. By (3.3) we have $(X, Y)_{\rho}=K_{\rho, 0}=S_{\theta, m, 0}(X, Y)$ with $\theta=2 m \rho /(1-\rho)$. Let $u \in(X, Y)_{\rho}$. By the very definition of $S_{\theta, m, 0}(X, Y)$ we may find a sequence $v_{n} \in X$ such that

$$
\pi_{\theta, m, 0}\left(u,\left(v_{n}\right)_{n}\right)=\sum_{n=1}^{\infty} 2^{n \theta}\left\|u-v_{n}\right\|_{Y}+\frac{1}{2^{2 n m}}\left\|v_{n}\right\|_{X}<\infty .
$$


For $u \in Y=\left(W^{k, p_{*}}\right)^{*}$ we define $T_{\delta}^{*} u$ by $\left\langle T_{\delta}^{*} u, \phi\right\rangle=\left\langle u, T_{\delta} \phi\right\rangle-$ the operators $T_{\delta}, T_{\delta}^{*}, \widetilde{T}_{\delta}$ are defined in the Step 1 of the proof of Theorem 2.3), see (2.29). The functional $T_{\delta}^{*} u$ is associated to the function $f(y):=\Phi_{\delta}(y)\left\langle u, \gamma_{\delta}(\cdot-y)\right\rangle$ in the sense that $\left\langle T_{\delta}^{*} u, \phi\right\rangle=$ $\int f(y) \phi(y) d y$.

Since $\left\|T_{\delta} \phi\right\|_{W^{k, p_{*}}} \leq\|\phi\|_{W^{k, p_{*}}}$ we obtain $\left\|T_{\delta}^{*} u-T_{\delta}^{*} v_{n}\right\|_{Y} \leq\left\|u-v_{n}\right\|_{Y}$. Notice that the notation $T_{\delta}^{*} v_{n}$ is introduced for the functional $\phi \rightarrow \int v_{n}(y) \phi(y) d y$. So $\left\langle T_{\delta}^{*} v_{n}, \phi\right\rangle=\left\langle f_{n}, \phi\right\rangle$ with $f_{n}(y)=\Phi_{\delta}(y)\left\langle v_{n}, \gamma_{\delta}(\cdot-y)\right\rangle=\Phi_{\delta}(y) v_{n} * \gamma_{\delta}(y)=\widetilde{T}_{\delta} v_{n}$. We have already proved in (2.31) (take $f_{n}=v_{n}$ ) that $\left\|\widetilde{T}_{\delta} v_{n}\right\|_{X} \leq\left\|v_{n}\right\|_{X}$. We conclude that

$$
\pi_{\theta, m, 0}\left(T_{\delta}^{*} u,\left(T_{\delta}^{*} v_{n}\right)_{n}\right) \leq \pi_{\theta, m, 0}\left(u,\left(v_{n}\right)_{n}\right)
$$

Using (2.28), b) we obtain

$$
\left\|T_{\delta}^{*} u\right\|_{W^{q, p}} \leq C \pi_{\theta, m, 0}\left(u,\left(v_{n}\right)_{n}\right)
$$

so the family $T_{\delta}^{*} u, \delta>0$ is bounded and consequently relatively compact in $W^{q, p}$. Let $\widetilde{u} \in W^{q, p}$ be a limit point. Since $\left\langle T_{\delta}^{*} u, \phi\right\rangle \rightarrow\langle u, \phi\rangle$ we have $\langle u, \phi\rangle=\langle\widetilde{u}, \phi\rangle$.

The proof of the inclusion $W^{q+1,2 m, e} \subset\left(W^{2 m+q, 2 m, p}, W^{-k, p_{*}}\right)_{\rho}$ is analogous to that of (6.5) so we skip it.

Remark 3.5 Let us compare this result with the one in Example 1 given before. We have replaced the dual of $W^{k, \infty}$ by the dual of $W^{k, p_{*}}$ and then the interpolation index is smaller and does no more depend on the dimension (because $d / p_{*}$ does no more appear).

\section{Diffusion processes}

We consider the SDE

$$
X_{t}=x+\sum_{j=1}^{N} \int_{0}^{t} \sigma_{j}\left(s, X_{s}\right) d W_{s}^{j}+\int_{0}^{t} b\left(s, X_{s}\right) d s
$$

with $\sigma_{j}, b: \mathbb{R}_{+} \times \mathbb{R}^{d} \rightarrow \mathbb{R}^{d}, j=1, \ldots, N$ measurable functions and $W$ a standard $N$ dimensional Brownian motion. We want to keep weak hypotheses on the coefficients so we do not know that the above SDE has a unique solution. So, we just consider a continuous and adapted process $X_{t}, t \geq 0$, which verifies (4.1). We assume that the coefficients have linear growth: for every $T>0$ there exists a constant $C_{T}$ such that

$$
|b(t, x)|+\sum_{j=1}^{N}\left|\sigma_{j}(t, x)\right| \leq C_{T}(1+|x|) \quad \forall(t, x) \in[0, T] \times \mathbb{R}^{d} .
$$

Moreover for an open domain $D \subset \mathbb{R}^{d}$ we define $C_{\log }([0, T] \times D)$ to be the set of functions $f:[0, T] \times D \rightarrow \mathbb{R}^{d}$ for which there exist $C, h>0$ such that for every $(t, x),(s, y) \in$ $[0, T] \times D$

$$
|f(t, x)-f(s, y)| \leq C\left(|\ln | x-\left.y\right|^{-(2+h)}+|\ln | t-\left.s\right|^{-(2+h)}\right) .
$$

Finally we say that a probability measure $\mu$ on $\mathbb{R}^{d}$ has a local density $p_{\mu}$ on $D$ if $\mu(A)=$ $\int_{A} p_{\mu}(x) d x$ for every Borel set $A \subset D$. We consider now an open domain $\Gamma \subset \mathbb{R}^{d}$ and we define

$$
\tau=\inf \left\{t: X_{t} \notin \Gamma\right\}
$$




\subsection{A local ellipticity condition}

We first study the case in which the diffusion coefficients $\sigma_{j}, j=1, \ldots, N$, satisfy a local ellipticity condition. More precisely, we prove that

Theorem 4.1 Let the coefficients $b$ and $\sigma_{j}, j=1, \ldots, N$, be measurable and with linear growth. For $y_{0} \in \Gamma, T>0$ and $0<r<\frac{1}{2} d\left(y_{0}, \Gamma^{c}\right)$, suppose that $\sigma_{j} \in C_{\log }([0, T] \times$ $\left.B_{r}\left(y_{0}\right)\right), j=1, \ldots, N$ and $\sigma \sigma^{*}\left(y_{0}\right)>0$. Let $X_{t}$ be any continuous and adapted solution to the SDE (4.1). Then the law of $X_{T \wedge \tau}$ has a local density on $B_{r / 4}\left(y_{0}\right)$.

We will need the following approximation result. We fix $\delta>0$ and we construct $X_{t}^{\delta}$ by

$$
X_{t}^{\delta}=X_{(T-\delta) \wedge \tau}+\sum_{j=1}^{N} \sigma\left(T-\delta, X_{T-\delta}\right)\left(W_{t}^{j}-W_{T-\delta}^{j}\right), \quad t \in[T-\delta, T] .
$$

We denote by $\mu$ the law of $X_{T \wedge \tau}$ and by $\mu_{\delta}$ the law of $X_{T}^{\delta}$. Moreover we consider a truncation function $\psi \in C^{\infty}$ such that $1_{B_{r / 2}\left(y_{0}\right)} \leq \psi \leq 1_{B_{r}\left(y_{0}\right)}$ and such that for every multi index $\alpha$ one has $\left\|\partial^{\alpha} \psi\right\|_{\infty} \leq C_{\alpha} r^{-|\alpha|}$ where $C_{\alpha}$ depends on $\alpha$ but not on $r$. We define $\nu=\psi \mu$ and $\nu_{\delta}=\psi \mu_{\delta}$.

Lemma 4.2 Under the hypothesis of the Theorem 4.1 one has

$$
d_{1}\left(\nu, \nu_{\delta}\right) \leq \frac{C_{*}}{r} \delta^{1 / 2}(\ln \delta)^{-(2+h)}
$$

where $C_{*}>0$ depends on the constants in (4.2) and (4.3) associated to $\sigma_{j}$ and $b, h$ being the constant from (4.3) related to $\sigma_{j}$ and $b$.

Proof. Since $X_{\tau} \in \Gamma^{c}$ and $r \leq \frac{1}{2} d\left(y_{0}, \Gamma^{c}\right)$ we have $\psi\left(X_{\tau}\right)=0$ so $\psi\left(X_{T \wedge \tau}\right)=\psi\left(X_{T}\right) 1_{\{\tau>T\}}$. It follows that for any $\phi \in C_{b}^{1}\left(\mathbb{R}^{d}\right)$

$$
\int \phi d \nu=\mathbb{E}\left(\phi \psi\left(X_{T \wedge \tau}\right)=\mathbb{E}\left(\phi \psi\left(X_{T}\right) 1_{\{\tau>T\}}\right)=R_{\delta}+I\right.
$$

with

$$
R_{\delta}=\mathbb{E}\left(\left(\phi \psi\left(X_{T}\right)-\phi \psi\left(X_{T}^{\delta}\right)\right) 1_{\{\tau>T\}}\right) \quad \text { and } \quad I=\mathbb{E}\left(\phi \psi\left(X_{T}^{\delta}\right) 1_{\{\tau>T\}}\right) .
$$

We write

$$
I=\int \phi d \nu_{\delta}-J \quad \text { with } \quad J=\mathbb{E}\left(\phi \psi\left(X_{T}^{\delta}\right) 1_{\{\tau \leq T\}}\right)
$$

So

$$
\left|\int \phi d \nu-\int \phi d \nu_{\delta}\right| \leq|J|+\left|R_{\delta}\right|
$$

We estimate $|J|$. Let $\eta=\inf \left\{t>T-\delta:\left|X_{t}-X_{(T-\delta) \wedge \tau}\right|>\frac{r}{4}\right\}$ and $\eta_{\delta}=\inf \{t>T-\delta$ : $\left.\left|X_{t}^{\delta}-X_{(T-\delta) \wedge \tau}\right|>\frac{r}{4}\right\}$. Using standard arguments one checks that

$$
\mathbb{P}(\eta<T)+\mathbb{P}\left(\eta_{\delta}<T\right) \leq C \delta
$$


Suppose that we are on the set $\{\eta>T\} \cap\left\{\eta_{\delta}>T\right\}$. If $\psi\left(X_{T}^{\delta}\right) \neq 0$ then $\left|X_{T}^{\delta}-y_{0}\right| \leq \frac{r}{2}$ and since $\eta_{\delta}>T$ it follows that $\left|X_{(T-\delta) \wedge \tau}-y_{0}\right| \leq \frac{3 r}{4}<r$. Since $\left|X_{\tau}-y_{0}\right|>r$ this implies that $\tau>T-\delta$. And we have $\left|X_{T-\delta}-y_{0}\right| \leq \frac{3 r}{4}$. And since $\eta>T$ it follows also that $\left|X_{t}-y_{0}\right| \leq r$ for every $T-\delta \leq t \leq T$. Then $X_{t} \in \Gamma$ and so $\tau>T$. We conclude that $\left\{\psi\left(X_{T}^{\delta}\right) \neq 0\right\} \cap\{\tau \leq T\} \subset\{\eta<T\} \cup\left\{\eta_{\delta}<T\right\}$. So that

$$
|J| \leq\|\phi \psi\|_{\infty}\left(\mathbb{P}(\eta<T)+\mathbb{P}\left(\eta_{\delta}<T\right)\right) \leq C\|\phi\|_{\infty} \delta .
$$

We estimate now

$$
\begin{aligned}
\left|R_{\delta}\right| & \leq\|\nabla(\phi \psi)\|_{\infty} \mathbb{E}\left(\left|X_{T}-X_{T}^{\delta}\right| 1_{\{\tau>T\}} 1_{\left\{X_{T} \in B_{r}\left(y_{0}\right)\right\}}\right) \\
& \leq \frac{C}{r}\left(\|\phi\|_{\infty}+\|\nabla \phi\|_{\infty}\right) \mathbb{E}\left(\left|X_{T}-X_{T}^{\delta}\right| 1_{\{\eta>T\} \cap\{\tau>T\} \cap\left\{X_{T} \in B_{r}\left(y_{0}\right)\right\}}\right)+C\|\phi\|_{\infty} \delta .
\end{aligned}
$$

On the set $\{\eta>T\} \cap\{\tau>T\} \cap\left\{X_{T} \in B_{r}\left(y_{0}\right)\right\}$ we have

$$
\begin{aligned}
X_{T}-X_{T}^{\delta}= & \sum_{j=1}^{N} \int_{T-\delta}^{T}\left(\sigma_{j}\left(t, X_{t}\right)-\sigma_{j}\left(T-\delta, X_{T-\delta}\right)\right) 1_{\left\{X_{T-\delta} \in B_{2 r}\left(y_{0}\right)\right\} \cap\left\{X_{t} \in B_{2 r}\left(y_{0}\right)\right\}} d W_{t}^{j}+ \\
& +\int_{T-\delta}^{T} b\left(t, X_{t}\right) d t .
\end{aligned}
$$

If we are on the set $\left\{X_{T-\delta} \in B_{2 r}\left(y_{0}\right)\right\} \cap\left\{X_{t} \in B_{2 r}\left(y_{0}\right)\right\}$ we may use (4.3) and we obtain

$$
\begin{aligned}
\left|\sigma_{j}\left(t, X_{t}\right)-\sigma_{j}\left(T-\delta, X_{T-\delta}\right)\right| \leq & C\left(1_{\left\{\left|X_{t}-X_{T-\delta}\right| \geq \delta^{-1 / 4}\right\}}+\right. \\
& \left.+|\ln | X_{t}-X_{T-\delta}||^{-(1+h)} 1_{\left\{\left|X_{t}-X_{T-\delta}\right|<\delta^{-1 / 4}\right\}}+\left|\ln \frac{1}{\delta}\right|^{-(2+h)}\right) \\
\leq & C 1_{\left\{\left|X_{t}-X_{T-\delta}\right| \geq \delta^{-1 / 4}\right\}}+2 C\left|\ln \frac{1}{\delta}\right|^{-(2+h)} .
\end{aligned}
$$

It follows that

$$
\begin{aligned}
& \mathbb{E}\left(\left|\int_{T-\delta}^{T}\left(\sigma_{j}\left(t, X_{t}\right)-\sigma_{j}\left(T-\delta, X_{T-\delta}\right)\right) d W_{t}^{j}\right|^{2} 1_{\{\eta>T\} \cap\{\tau>T\} \cap\left\{X_{T} \in B_{r}\left(y_{0}\right)\right\}}\right) \\
\leq & \int_{T-\delta}^{T} \mathbb{E}\left(\left|\sigma_{j}\left(t, X_{t}\right)-\sigma_{j}\left(T-\delta, X_{T-\delta}\right)\right|^{2} 1_{\left\{X_{T-\delta} \in B_{2 r}\left(y_{0}\right)\right\} \cap\left\{X_{t} \in B_{2 r}\left(y_{0}\right)\right\}}\right) d t \\
\leq & C \delta\left|\ln \frac{1}{\delta}\right|^{-2(2+h)}+\int_{T-\delta}^{T} \mathbb{P}\left(\left|X_{t}-X_{T-\delta}\right| \geq \delta^{-1 / 4}\right) d t \leq C \delta\left|\ln \frac{1}{\delta}\right|^{-2(2+h)}+C \delta^{2} .
\end{aligned}
$$

The drift term may be upper bounded directly:

$$
\mathbb{E}\left(\left|\int_{T-\delta}^{T} b\left(t, X_{t}\right) d t\right|^{2}\right) \leq C \mathbb{E}\left(\left|\int_{T-\delta}^{T}\left(1+\left|X_{t}\right|\right) d t\right|^{2}\right) \leq C \delta^{2} .
$$


So we have proved that

$$
\left|R_{\delta}\right| \leq \frac{C}{r}\left(\|\phi\|_{\infty}+\|\nabla \phi\|_{\infty}\right) \frac{\delta^{1 / 2}}{|\ln \delta|^{2+h}}
$$

and finally

$$
\left|\int \phi d \nu-\int \phi d \nu_{\delta}\right| \leq \frac{C}{r}\left(\|\phi\|_{\infty}+\|\nabla \phi\|_{\infty}\right) \frac{\delta^{1 / 2}}{|\ln \delta|^{2+h}}
$$

Proof of Theorem 4.1. Step 1. We define $\bar{\nu}$ and $\bar{\nu}_{\delta}$ by

$$
\int \phi d \bar{\nu}=\mathbb{E}\left(\phi \psi\left(X_{T \wedge \tau}\right) 1_{\left\{X_{T-\delta} \in B_{2 r}\left(y_{0}\right)\right\}}\right) \quad \text { and } \quad \int \phi d \bar{\nu}_{\delta}=\mathbb{E}\left(\phi \psi\left(X_{T}^{\delta}\right) 1_{\left\{X_{T-\delta} \in B_{2 r}\left(y_{0}\right)\right\}}\right) .
$$

One has

$$
\left|\int \phi d \bar{\nu}-\int \phi d \nu\right| \leq\|\phi \psi\|_{\infty} \mathbb{P}\left(\left\{X_{T} \in B_{r}\left(y_{0}\right)\right\} \cap\left\{X_{T-\delta} \in B_{2 r}^{c}\left(y_{0}\right)\right\}\right) \leq C\|\phi \psi\|_{\infty} \delta
$$

so that $d_{1}(\nu, \bar{\nu}) \leq C \delta$. In the same way $d_{1}\left(\nu_{\delta}, \bar{\nu}_{\delta}\right) \leq C \delta$ so that, using the previous lemma

$$
d_{1}\left(\nu, \bar{\nu}_{\delta}\right) \leq \frac{C}{r} \delta^{1 / 2}(\ln \delta)^{-(2+h)}
$$

Step 2. We have $\bar{\nu}_{\delta}(d y)=p_{\delta}(y) d y$ with

$$
p_{\delta}(y)=\mathbb{E}\left(\psi(y) \gamma_{a_{\delta}\left(X_{T \wedge \tau}\right)}\left(y-X_{T \wedge \tau}\right) 1_{\left\{X_{T-\delta} \in B_{2 r}\left(y_{0}\right)\right\}}\right)
$$

where $a_{\delta}(x)=\delta \sigma \sigma^{*}(x)$ and

$$
\gamma_{a_{\delta}(x)}(y)=\frac{1}{\sqrt{\operatorname{det} a_{\delta}(x)}} \exp \left(-\left\langle a_{\delta}^{-1}(x) y, y\right\rangle\right)
$$

is the Gaussian density of covariance matrix $a_{\delta}(x)$. We may find a constant $\lambda_{*}$ such that the lower eigenvalue of $a_{\delta}(x)$ is larger then $\delta \lambda_{*}$ for $x \in B_{2 r}\left(y_{0}\right)$. It is then easy to check that for every multi index $\alpha$ one has

$$
\sup _{x \in B_{2 r}\left(y_{0}\right)}\left\|\partial^{\alpha} \gamma_{a_{\delta}(x)}\right\|_{\infty} \leq C \delta^{-|\alpha| / 2} .
$$

We also have $\left\|\partial^{\alpha} \psi\right\|_{\infty} \leq C r^{-|\alpha|}$ so it is easy to check that

$$
\left\|p_{\delta}\right\|_{2 m+q, 2 m, p} \leq C\left(r, y_{0}\right) \times \delta^{-\frac{1}{2}(2 m+q)} \quad \text { with } \quad C\left(r, y_{0}\right)=\frac{C\left|y_{0}\right|^{2 m}}{r^{2 m+q}} .
$$

Step 3. We will use the criterion (2.9) so we focus on $e_{\log }$. And we have for $p>1$

$$
\left\|p_{\delta}\right\|_{2 m+q, 2 m, e_{\log }} \leq\left\|p_{\delta}\right\|_{2 m+q, 2 m, p} \leq C\left(r, y_{0}\right) \times \delta^{-\frac{1}{2}(2 m+q)} .
$$


We conclude that

$$
p_{\delta} \in M_{m, q, e_{\log }}\left(R_{\delta}\right) \quad \text { with } \quad R_{\delta}=C\left(r, y_{0}\right) \times \delta^{-\frac{1}{2}(2 m+q)} .
$$

We have $\delta^{1 / 2}=C R_{\delta}^{1 /(2 m+q)}$ so (4.4) reads

$$
d_{1}\left(\nu, M_{m, q, e_{\log }}\left(R_{\delta}\right)\right) \leq \frac{C R_{\delta}^{1 /(2 m+q)}}{\left(\ln R_{\delta}\right)^{2+h}} .
$$

Now we look to (2.9) with $k=1$ and $a>1$ :

$$
R_{\delta}^{\frac{q+1}{2 m}}\left(\ln R_{\delta}\right)^{a\left(1+\frac{q+1}{2 m}\right)+1} d_{1}\left(\nu, M_{m, q, e_{\log }}\left(R_{\delta}\right)\right) \leq R_{\delta}^{\frac{q+1}{2 m}-\frac{1}{2 m+q}}\left(\ln R_{\delta}\right)^{a\left(1+\frac{q+1}{2 m}\right)-1-h}
$$

It is clear that if $q>0$ the above quantity blows up, but, if $q=0$ then $\frac{q+1}{2 m}-\frac{1}{2 m+q}=0$ and this term disappears. Moreover we take $m$ sufficiently large in order to have $\frac{1}{2 m}<h$ and $a>1$ sufficiently small in order to have $a\left(1+\frac{1}{2 m}\right) \leq 1+h$. And then the above quantity is bounded. So $\nu$ is absolutely continuous and then $\mu$ is absolutely continuous on $B_{r}\left(y_{0}\right)$.

\subsection{A local Hörmander condition}

In this section we work under a local Hörmander condition. In contrast with the situation from the previous section in this case we are no more able to exhibit an explicit density (as the non degenerated Gaussian density in the previous section) so we are obliged to use integration by parts. This is the specific point in this example.

In this framework it is difficult to work with time dependent coefficients so now on $\sigma_{j}, b$ depend on $x$ only. Our equation is

$$
X_{t}=x+\sum_{j=1}^{N} \int_{0}^{t} \sigma_{j}\left(X_{s}\right) d W_{s}^{j}+\int_{0}^{t} b\left(X_{s}\right) d s .
$$

We denote by $\sigma_{0}$ the drift coefficient when writing the equation in Stratonovich form that is $\sigma_{0}=b-\frac{1}{2} \sum_{j=1}^{N} \sum_{k=1}^{d} \sigma_{j}^{k} \partial_{k} \sigma_{j}$. We can do this only if $\sigma_{j}$ are one time differentiable - in our case this will be locally true, and this will be sufficient. For an open domain $D \subset \mathbb{R}^{d}$ we denote by $C^{k}(D)$ the class of functions which are $k$ time differentiable on $D$. For $l \leq k$ we construct recursively

$$
\mathcal{A}_{0}=\left\{\sigma_{j}, j=1, \ldots, N\right\}, \quad \mathcal{A}_{l}=\left\{\left[\phi, \sigma_{j}\right], \phi \in \mathcal{A}_{l-1}, j=0, \ldots, N\right\}
$$

and

$$
\Lambda_{k}(\sigma, x)=\inf _{|\xi|=1} \sum_{\phi \in \cup_{l=0}^{k} \mathcal{A}_{l}}\langle\phi(x), \xi\rangle^{2} .
$$

So the condition $\Lambda_{0}(\sigma, x)>0$ says that we have ellipticity in the point $x$ and the condition $\Lambda_{k}(\sigma, x)>0$ says that the weak Hörmander condition of order $k$ holds in $x$. 
Theorem 4.3 Let $\Gamma \subset \mathbb{R}^{d}$ be an open domain and $\tau$ be the exit time from $\Gamma$. Fix $y_{0} \in \Gamma$ and $r<\frac{1}{2} d\left(y_{0}, \Gamma^{c}\right)$. Suppose that the diffusion coefficients have linear growth, $\sigma_{j}, b \in C^{k}\left(B_{r}\left(y_{0}\right)\right), j=0,1, \ldots, N$, for some $k \geq 2$ and moreover, $\Lambda_{k}(\sigma, x) \geq \varepsilon_{0}>0$, for $x \in B_{r}\left(y_{0}\right)$. Then for every $T>0$ the law $\mu$ of $X_{T \wedge \tau}$ is absolutely continuous with respect to the Lebesgue measure with density $p_{\mu} \in C^{k-2}\left(B_{r / 4}\left(y_{0}\right)\right)$.

Remark 4.4 In Proposition 23 from [1] we prove a similar result for a diffusion process with coefficients which are globally in $C^{k}\left(\mathbb{R}^{d}\right)$ and for which we do not consider the stopping time $\tau$. Here we generalize this result for local regularity and with a stopping time.

The proof is analogous to the proof of the previous theorem but now we will use another approximation process. For $\delta>0$ we consider some coefficients $\sigma_{j}^{\delta}, b^{\delta} \in C^{k}\left(\mathbb{R}^{d}\right), j=$ $1, \ldots, N$ such that $\sigma_{j}^{\delta}(y)=\sigma_{j}(y), b^{\delta}(y)=b(y)$ for $y \in B_{r}\left(y_{0}\right)$ and such that

$$
\sum_{0 \leq|\alpha| \leq k}\left\|\partial^{\alpha} \sigma_{j}^{\delta}\right\|_{\infty} \leq \sum_{0 \leq|\alpha| \leq k}\left\|1_{B_{r}\left(y_{0}\right)} \partial^{\alpha} \sigma_{j}\right\|_{\infty}
$$

for $j=1, \ldots, N$ and the same for $b^{\delta}$. We also assume that

$$
\inf _{x \in \mathbb{R}^{N}} \Lambda_{k}\left(\sigma^{\delta}, x\right) \geq \inf _{x \in B_{r}\left(y_{0}\right)} \Lambda_{k}(\sigma, x)=: \varepsilon_{*}>0
$$

Then we define $X_{t}^{\delta}=X_{t}$ for $t \leq T-\delta$ and

$$
X_{t}^{\delta}=X_{(T-\delta) \wedge \tau}+\sum_{j=1}^{N} \int_{(T-\delta) \wedge \tau_{\Gamma}}^{t} \sigma_{j}^{\delta}\left(X_{s}^{\delta}\right) d W_{s}^{j}+\int_{(T-\delta) \wedge \tau_{\Gamma}}^{t} b\left(X_{s}^{\delta}\right) d s \quad \text { for }(T-\delta) \wedge \tau \leq t \leq T .
$$

We denote by $\mu$ the law of $X_{T \wedge \tau}$ and by $\mu_{\delta}$ the law of $X_{T}^{\delta}$. Moreover we consider a truncation function $\psi \in C^{\infty}$ such that $1_{B_{r / 2}\left(y_{0}\right)} \leq \psi \leq 1_{B_{r}\left(y_{0}\right)}$ and such that for every multi index $\alpha$ and every $p \geq 1$ there exists a constant $C_{\alpha, p}$ such that

$$
\left|\frac{\partial^{\alpha} \psi}{\psi}(x)\right|^{p} \times \psi(x) \leq \frac{C_{\alpha, p}}{r^{|\alpha|}} .
$$

Such a function is constructed for example in [1], Section 2.7. We define $\nu=\psi \mu$ and $\nu_{\delta}=\psi \mu_{\delta}$.

Lemma 4.5 Under the hypotheses of Theorem 4.3, for every $p>1$ one has

$$
d_{1}\left(\nu, \nu_{\delta}\right) \leq \frac{C_{p}}{r^{p}} \delta^{p / 2}
$$

Proof. We define $\eta=\inf \left\{t \geq T-\delta:\left|X_{t}-X_{T-\delta}\right|>\frac{r}{4}\right\}, \eta_{\delta}=\inf \{t \geq T-\delta$ : $\left.\left|X_{t}^{\delta}-X_{T-\delta}^{\delta}\right|>\frac{r}{4}\right\}$ and we write

$$
\begin{aligned}
& \left|\int \phi d \nu-\int \phi d \nu_{\delta}\right| \leq I+2\|\phi\|_{\infty}\left(\mathbb{P}(\eta \leq T)+\mathbb{P}\left(\eta_{\delta} \leq T\right)\right) \quad \text { with } \\
& I=\mathbb{E}\left(\left|\phi \psi\left(X_{T \wedge \tau}\right)-\phi \psi\left(X_{T}^{\delta}\right)\right| 1_{\{\eta>T\} \cap\left\{\eta_{\delta}>T\right\}}\right) .
\end{aligned}
$$


Since the coefficients $\sigma_{j}$ have linear growth we have for every $p$

$$
\mathbb{P}(\eta \leq T) \leq \mathbb{P}\left(\sup _{T-\delta \leq t \leq T}\left|X_{t}(x)-X_{T-\delta}(x)\right| \geq \frac{r}{2}\right) \leq \frac{C_{p}}{r^{p}} \delta^{p / 2} .
$$

And the same is true for $\mathbb{P}\left(\eta_{\delta} \leq T\right)$.

We will now check that $I=0$. We write

$$
\begin{aligned}
I & =I^{\prime}+I^{\prime \prime} \quad \text { with } \\
I^{\prime} & =\mathbb{E}\left(\left|\phi \psi\left(X_{T \wedge \tau}\right)-\phi \psi\left(X_{T}^{\delta}\right)\right| 1_{\{\eta>T\} \cap\left\{\eta_{\delta}>T\right\}} 1_{\{\tau<T}\right), \\
I^{\prime \prime} & =\mathbb{E}\left(\left|\phi \psi\left(X_{T \wedge \tau}\right)-\phi \psi\left(X_{T}^{\delta}\right)\right| 1_{\{\eta>T\} \cap\left\{\eta_{\delta}>T\right\}} 1_{\{\tau>T}\right) .
\end{aligned}
$$

We have $\psi\left(X_{\tau}\right)=0$. Suppose now that $\psi\left(X_{T}^{\delta}\right) \neq 0$ and $\eta_{\delta}>T$. Then $X_{(T-\delta) \wedge \tau} \in$ $B_{3 r / 4}\left(y_{0}\right) \subset \Gamma$ so $\tau>T-\delta$. But then, since $\eta>T$ it follows that $\tau>T$ which is in contradiction with $\tau<T$. So we have proved that $I^{\prime}=0$.

We check now that $I^{\prime \prime}=0$. We are on the set $\tau>T$ so $\tau$ disappears from the equation of $X_{t}^{\delta}$. We are also on the set $\{\eta>T\}$. If $\psi\left(X_{T}(x)\right) \neq 0$ it follows that $X_{T} \in B_{r / 4}\left(y_{0}\right)$ and consequently for every $T-\delta \leq t \leq T$ we have $X_{t} \in B_{r / 2}\left(y_{0}\right)$. On this set the coefficients $\sigma_{j}$ and $\sigma_{j}^{\delta}$ coincide so $X_{t}$ and $X_{t}^{\delta}$ solve the same equation. Since the coefficients are Lipschitz continuous on $B_{r / 2}\left(y_{0}\right)$ this equation has the uniqueness property. We conclude that $X_{t}=X_{t}^{\delta}$ for $T-\delta \leq t \leq T$. Suppose now that $\psi\left(X_{T}^{\delta}\right) \neq 0$. Then, since we are on the set $\left\{\eta_{\delta}>T\right\}$, the same reasoning gives that $X_{t}=X_{t}^{\delta}$ for $T-\delta \leq t \leq T$. So $I^{\prime \prime}=0$ and we obtain

$$
\left|\int \phi d \nu-\int \phi d \nu_{\delta}\right| \leq \frac{C_{p}}{r^{p}}\|\phi\|_{\infty} \delta^{p / 2}
$$

We now prove the following estimate for the Sobolev norms of $\nu_{\delta}$ :

Lemma 4.6 Under the hypotheses of the Theorem 4.3, for every $q \leq k-1$ and $p>1$ there exist $C_{q, p}$ and $l_{q}$ such that

$$
\|1\|_{W_{\nu_{\delta}}^{q, p}} \leq C_{q, p} \delta^{-l_{q}}
$$

Proof. We consider the semigroup associated to the diffusion process $X_{t}$ that is $P_{t} f(x)=$ $\mathbb{E}\left(f\left(X_{t}(x)\right)\right)$ where $X_{t}(x)$ is the solution to our SDE starting from $X_{0}=x$. And similarly $P_{t}^{\delta} f(x)=\mathbb{E}\left(f\left(X_{t}^{\delta}(x)\right)\right)$. Then

$$
\int f d \nu_{\delta}=\mathbb{E}\left(f\left(X_{T}^{\delta}\right) \psi\left(X_{T}^{\delta}\right)\right)=\int P_{T-\delta}(x, d y) \int f(z) \psi(z) P_{\delta}^{\delta}(y, d z) .
$$

A standard result concerning diffusion processes under Hörmander conditions (see [17]) says that for every multi index $\alpha$

$$
\mathbb{E}\left(\psi\left(X_{\delta}^{\delta}(x)\right) \partial^{\alpha} f\left(X_{\delta}^{\delta}(x)\right)=\mathbb{E}\left(f\left(X_{\delta}^{\delta}\right) H_{\alpha}\left(X_{\delta}^{\delta}(x), \psi\left(X_{\delta}^{\delta}(x)\right)\right)\right)\right.
$$

where $H_{\alpha}\left(X_{\delta}^{\delta}(x), \psi\left(X_{\delta}^{\delta}(x)\right)\right)$ is the weight which appears in the integration by parts formula using Malliavin calculus. An inspection of the structure of this weight shows that

$$
H_{\alpha}\left(X_{\delta}^{\delta}(x), \psi\left(X_{\delta}^{\delta}(x)\right)=\sum_{|\beta| \leq|\alpha|} \partial^{\beta} \psi\left(X_{\delta}^{\delta}(x)\right) \theta_{\beta}(x)\right.
$$


where $\theta_{\beta}(x)$ is built using Malliavin derivatives of $X_{\delta}^{\delta}(x)$, the Ornstein Uhlembeck operator and the inverse of the covariance matrix of $X_{\delta}^{\delta}(x)$. Moreover, under the uniform Hörmander condition (4.7) and the boundedness condition (4.6) one can see that for every $p \geq 1$

$$
\mathbb{E}\left|\theta_{\beta}(x)\right|^{p} \leq \frac{C}{\delta^{l}}
$$

where $C$ depends on $p$, on $\varepsilon_{0}$ and on the bounds in (4.6) and $l \in \mathbb{N}$ is a power which depends on the order of the Hörmander condition. Moreover, taking conditional expectations we obtain

$$
\begin{aligned}
\mathbb{E}\left(f\left(X_{\delta}^{\delta}(x)\right) H_{\alpha}\left(X_{\delta}^{\delta}(x), \psi\left(X_{\delta}^{\delta}(x)\right)\right)\right) & =\mathbb{E}\left(f\left(X_{\delta}^{\delta}(x)\right) \mathbb{E}\left(H_{\alpha}\left(X_{\delta}^{\delta}(x), \psi\left(X_{\delta}^{\delta}(x)\right) \mid X_{\delta}^{\delta}(x)\right)\right)\right. \\
& =\sum_{|\beta| \leq|\alpha|} \mathbb{E}\left(f\left(X_{\delta}^{\delta}(x)\right) \partial^{\beta} \psi\left(X_{\delta}^{\delta}(x)\right) \mathbb{E}\left(\theta_{\beta}(x) \mid X_{\delta}^{\delta}(x)\right)\right) \\
& =\sum_{|\beta| \leq|\alpha|} \mathbb{E}\left(f\left(X_{\delta}^{\delta}(x)\right) \partial^{\beta} \psi\left(X_{\delta}^{\delta}(x)\right) \widehat{\theta}_{\beta}\left(X_{\delta}^{\delta}(x)\right)\right)
\end{aligned}
$$

where $\widehat{\theta}_{\beta}\left(X_{\delta}^{\delta}(x)\right)=\mathbb{E}\left(\theta_{\beta}(x) \mid X_{\delta}^{\delta}(x)\right)$. Using Hölder inequality

$$
\mathbb{E}\left|\widehat{\theta}_{\beta}(x)\right|^{p} \leq \mathbb{E}\left|\theta_{\beta}(x)\right|^{p} \leq \frac{C}{\delta^{l}} .
$$

We now come back and we write

$$
\begin{aligned}
\int \partial^{\alpha} f d \nu_{\delta} & =\mathbb{E}\left(\partial^{\alpha} f\left(X_{T}^{\delta}\right) \psi\left(X_{T}^{\delta}\right)\right)=\int P_{T-\delta}(x, d y) \int \partial^{\alpha} f(z) \psi(z) P_{\delta}^{\delta}(y, d z) \\
& =\int P_{T-\delta}(x, d y) \mathbb{E}\left(\partial^{\alpha} f\left(X_{\delta}^{\delta}(y)\right) \psi\left(X_{\delta}^{\delta}(y)\right)\right) \\
& \left.=\sum_{|\beta| \leq|\alpha|} \int P_{T-\delta}(x, d y) \mathbb{E}\left(f\left(X_{\delta}^{\delta}(y)\right) \partial^{\beta} \psi\left(X_{\delta}^{\delta}(y)\right) \widehat{\theta}_{\beta}\left(X_{\delta}^{\delta}(y)\right)\right)\right) \\
& \left.=\sum_{|\beta| \leq|\alpha|} \int P_{T-\delta}(x, d y) \mathbb{E}\left(f\left(X_{\delta}^{\delta}(y)\right) \frac{\partial^{\beta} \psi\left(X_{\delta}^{\delta}(y)\right)}{\psi\left(X_{\delta}^{\delta}(y)\right)} \widehat{\theta}_{\beta}\left(X_{\delta}^{\delta}(y)\right) \psi\left(X_{\delta}^{\delta}(y)\right)\right)\right) \\
& =\sum_{|\beta| \leq|\alpha|} \int P_{T-\delta}(x, d y) \int f(z) \frac{\partial^{\beta} \psi(z)}{\psi(z)} \widehat{\theta}_{\beta}(z) \psi(z) P_{\delta}^{\delta}(y, d z) \\
& =\int f(z)\left(\sum_{|\beta| \leq|\alpha|} \frac{\partial^{\beta} \psi(z)}{\psi(z)} \widehat{\theta}_{\beta}(z)\right) d \nu_{\delta}(z) .
\end{aligned}
$$

This proves that

$$
\partial_{\alpha}^{\nu_{\delta}} 1(z)=\sum_{|\beta| \leq|\alpha|} \frac{\partial^{\beta} \psi(z)}{\psi(z)} \widehat{\theta}_{\beta}(z) .
$$


Now we compute

$$
\begin{aligned}
\int\left|\partial_{\alpha}^{\nu_{\delta}} 1(z)\right|^{p} d \nu_{\delta}(z) & \leq C \sum_{|\beta| \leq|\alpha|} \int\left|\frac{\partial^{\beta} \psi(z)}{\psi(z)} \widehat{\theta}_{\beta}(z)\right|^{p} d \nu_{\delta}(z) \\
& =C \sum_{|\beta| \leq|\alpha|} \int\left|\frac{\partial^{\beta} \psi(z)}{\psi(z)} \widehat{\theta}_{\beta}(z)\right|^{p} \psi(z) d \mu_{\delta}(z) \\
& \leq C \sum_{|\beta| \leq|\alpha|} \int\left|\widehat{\theta}_{\beta}(z)\right|^{p} d \mu_{\delta}(z)
\end{aligned}
$$

last inequality being a consequence of the property (4.8) for $\psi$. And by (4.9)

$$
\int\left|\widehat{\theta}_{\beta}(z)\right|^{p} d \mu_{\delta}(z)=\mathbb{E}\left|\widehat{\theta}_{\beta}(x)\right|^{p} \leq \frac{C}{\delta^{l}} .
$$

Proof of Theorem 4.3. Since $\nu$ coincides with $\mu$ on $B_{r / 2}\left(y_{0}\right)$ we may look to the regularity of $\nu$ (instead of $\mu$ ). We recall now the hypothesis $\widetilde{H}_{q}(k, m, e$ ) with $k=1$ (we work with $\left.d_{1}\right), m=1$ and $e(t)=t^{p}$-in particular we have $\beta_{e_{p}}(t)=t^{1 / p_{*}}$ where $p_{*}$ is the conjugate of $p$. Then, we consider hypothesis

$$
\widetilde{H}_{q}\left(1,1, e_{p}\right): \varlimsup_{R \rightarrow \infty} \frac{L_{a}(R)^{1+\frac{1+q}{2}} L_{a}(R)^{\frac{d}{2 p *}}}{R} d_{1}\left(\nu, \widetilde{M}_{1, q, e_{p}}(R)\right)<\infty .
$$

We also recall that $L_{a}(R)=R(\ln R)^{a}$ for $a>1$ so that

$$
\frac{L_{a}(R)^{1+\frac{1+q}{2}} L_{a}(R)^{\frac{d}{2 p_{*}}}}{R}=R^{\frac{1+q}{2}+\frac{d}{2 p_{*}}}(\ln R)^{a\left(1+\frac{1+q}{2}+\frac{d}{2 p_{*}}\right)} .
$$

We also recall that $\widetilde{M}_{1, q, e_{p}}(R)$ is the class of probability measures $\rho$ such that $\|1\|_{W_{\rho}^{q, p}} \leq R$. So in the previous lemmas we have proved that $\nu_{\delta} \in \widetilde{M}_{1, q, e_{p}}\left(R_{\delta}\right)$ with $R_{\delta}=\delta^{-l_{q}}$ and $d_{1}\left(\nu, \nu_{\delta}\right) \leq C \delta^{n}=R_{\delta}^{-n / l_{q}}$ for some $l_{q}$ depending on $q$ and every $n \in \mathbb{N}$. So we have

$$
\frac{L_{a}(R)^{1+\frac{1+q}{2}} L_{a}(R)^{\frac{d}{2 p_{*}}}}{R} d_{1}\left(\nu, \widetilde{M}_{1, q, e_{p}}(R)\right) \leq R^{\frac{1+q}{2}+\frac{d}{2 p_{*}}}(\ln R)^{a\left(1+\frac{1+q}{2}+\frac{d}{2 p_{*}}\right)} \times R^{-n / l_{q}}
$$

and this inequality is true for every $n \in \mathbb{N}$. So, by Theorem 2.17 we have $\nu(d x)=p_{\nu}(x) d x$ with $p_{\nu} \in W^{k-1, p}$ for every $p \geq 1$. Then using the Sobolev embedding theorem we obtain that $p_{\nu} \in C^{k-2}$.

\section{Stochastic heat equation}

In this section we investigate the regularity of the law of the solution to the stochastic heat equation introduced by Walsh in [28]. Formally this equation is

$$
\partial_{t} u(t, x)=\partial_{x}^{2} u(t, x)+\sigma(u(t, x)) W(t, x)+b(u(t, x))
$$


where $W$ denotes a white noise on $\mathbb{R}_{+} \times[0,1]$. We consider Neumann boundary conditions that is $\partial_{x} u(t, 0)=\partial_{x} u(t, 1)=0$ and the initial condition is $u(0, x)=u_{0}(x)$. The rigorous formulation to this equation is given by the mild form constructed as follows. Let $G_{t}(x, y)$ be the fundamental solution to the deterministic heat equation $\partial_{t} v(t, x)=\partial_{x}^{2} v(t, x)$ with Neuman boundary conditions. Then $u$ satisfies

$$
\begin{aligned}
u(t, x)= & \int_{0}^{1} G_{t}(x, y) u_{0}(y) d y+\int_{0}^{t} \int_{0}^{1} G_{t-s}(x, y) \sigma(u(s, y)) d W(s, y) \\
& +\int_{0}^{t} \int_{0}^{1} G_{t-s}(x, y) b(u(s, y)) d y d s
\end{aligned}
$$

where $d W(s, y)$ is the Itô integral introduced by Walsh. The function $G_{t}(x, y)$ is explicitly known (see [28] or [5]) but here we will use just few properties that we list below (see the appendix in [5] for the proof). More precisely, for $0<\varepsilon<t$ we have

$$
\int_{t-\varepsilon}^{t} \int_{0}^{1} G_{t-s}^{2}(x, y) d y d s \leq C \varepsilon^{1 / 2}
$$

Moreover, for $0<x_{1}<\ldots<x_{d}<1$ there exists a constant $C$ depending on $\min _{i=1, d}\left(x_{i}-\right.$ $\left.x_{i-1}\right)$ such that

$$
C \varepsilon^{1 / 2} \geq \inf _{|\xi|=1} \int_{t-\varepsilon}^{t} \int_{0}^{1}\left(\sum_{i=1}^{d} \xi_{i} G_{t-s}\left(x_{i}, y\right)\right)^{2} d y d s \geq C^{-1} \varepsilon^{1 / 2} .
$$

This is an easy consequence of the inequalities (A2) and (A3) from [5].

In [23] one gives sufficient conditions in order to obtain the absolute continuity of the law of $u(t, x)$ for $(t, x) \in(0, \infty) \times[0,1]$ and in [5], under appropriate hypothesis, one obtains a $C^{\infty}$ density for the law of the vector $\left(u\left(t, x_{1}\right), \ldots, u\left(t, x_{d}\right)\right)$ with $\left(t, x_{i}\right) \in(0, \infty) \times\{\sigma \neq$ $0\}, i=1, \ldots, d$. The aim of this section is to obtain the same type of results but under much weaker regularity hypothesis on the coefficients. One may first discuss the absolute continuity of the law and further, under more regularity hypothesis on the coefficients, one may discuss the regularity of the density. Here, in order to avoid technicalities, we restrict ourself to the absolute continuity property. We also assume global ellipticity that is

$$
\sigma(x) \geq c_{\sigma}>0 \quad \text { for every } x \in[0,1] .
$$

A local ellipticity condition may also be used but again, this gives more technical complications that we want to avoid. This is somehow a benchmark for the efficiency of the method developed in the previous sections.

We assume the following regularity hypothesis: $\sigma, b$ are measurable and bounded functions and there exists $h>0$ such that

$$
|\sigma(x)-\sigma(y)| \leq|\ln | x-\left.y\right|^{-(2+h)}, \quad \text { for every } x, y \in[0,1] .
$$

This hypothesis is not sufficient in order to ensure existence and uniqueness for the solution to (5.2) (one needs $\sigma$ and $b$ to be globally Lipschitz continuous in order to obtain it) - so in the following we will just consider a random field $u(t, x),(t, x) \in(0, \infty) \times[0,1]$ which is adapted to the filtration generated by $W$ (see Walsh [28] for precise definitions) and which solves (5.2). 
Proposition 5.1 Suppose that (5.5) and (5.6) hold. Then for every $0<x_{1}<\ldots<x_{d}<1$ and $T>0$, the law of the random vector $U=\left(u\left(T, x_{1}\right), \ldots u\left(T, x_{d}\right)\right)$ is absolutely continuous with respect to the Lebesgue measure.

Proof. Given $0<\varepsilon<T$ we decompose

$$
u(T, x)=u_{\varepsilon}(T, x)+I_{\varepsilon}(T, x)+J_{\varepsilon}(T, x)
$$

with

$$
\begin{aligned}
u_{\varepsilon}(T, x)= & \int_{0}^{1} G_{t}(x, y) u_{0}(y) d y+\int_{0}^{T} \int_{0}^{1} G_{T-s}(x, y) \sigma(u(s \wedge(T-\varepsilon), y)) d W(s, y) \\
& +\int_{0}^{T-\varepsilon} \int_{0}^{1} G_{T-s}(x, y) b(u(s, y)) d y d s, \\
I_{\varepsilon}(T, x)= & \int_{T-\varepsilon}^{T} \int_{0}^{1} G_{T-s}(x, y)(\sigma(u(s, y))-\sigma(u(s \wedge(T-\varepsilon), y))) d W(s, y), \\
J_{\varepsilon}(T, x)= & \int_{T-\varepsilon}^{T} \int_{0}^{1} G_{T-s}(x, y) b(u(s, y)) d y d s .
\end{aligned}
$$

Step 1. We prove that

$$
\mathbb{E}\left|I_{\varepsilon}(T, x)\right|^{2}+\mathbb{E}\left|J_{\varepsilon}(T, x)\right|^{2} \leq C|\ln \varepsilon|^{-2(2+h)} \varepsilon^{1 / 2} .
$$

Let $\mu$ and $\mu_{\varepsilon}$ be the law of $U=\left(u\left(T, x_{1}\right), \ldots, u\left(T, x_{d}\right)\right)$ and $U_{\varepsilon}=\left(u_{\varepsilon}\left(T, x_{1}\right), \ldots, u_{\varepsilon}\left(T, x_{d}\right)\right)$ respectively. Using the above estimate one easily obtains

$$
d_{1}\left(\mu, \mu_{\varepsilon}\right) \leq C|\ln \varepsilon|^{-(2+h)} \varepsilon^{1 / 4} .
$$

Using the isometry property

$$
\mathbb{E}\left|I_{\varepsilon}(T, x)\right|^{2}=\int_{T-\varepsilon}^{T} \int_{0}^{1} G_{T-s}^{2}(x, y) \mathbb{E}\left(\sigma(u(s, y)-\sigma(u(s \wedge(T-\varepsilon), y)))^{2}\right) d y d s .
$$

We consider the set $\Lambda_{\varepsilon, \eta}(s, y)=\{|u(s, y)-u(s \wedge(T-\varepsilon), y)| \leq \eta\}$ and we split the above term as $\mathbb{E}\left|I_{\varepsilon}(T, x)\right|^{2}=A_{\varepsilon, \eta}+B_{\varepsilon, \eta}$ with

$$
\begin{aligned}
& A_{\varepsilon}=\int_{T-\varepsilon}^{T} \int_{0}^{1} G_{T-s}^{2}(x, y) \mathbb{E}\left(\sigma(u(s, y)-\sigma(u(s \wedge(T-\varepsilon), y)))^{2} 1_{\Lambda_{\varepsilon, \eta}(s, y)}\right) d y d s \\
& B_{\varepsilon}=\int_{T-\varepsilon}^{T} \int_{0}^{1} G_{T-s}^{2}(x, y) \mathbb{E}\left(\sigma(u(s, y)-\sigma(u(s \wedge(T-\varepsilon), y)))^{2} 1_{\Lambda_{\varepsilon, \eta}^{c}(s, y)}\right) d y d s .
\end{aligned}
$$

Using (5.6)

$$
A_{\varepsilon} \leq C(\ln \eta)^{2(2+h)} \int_{T-\varepsilon}^{T} \int_{0}^{1} G_{T-s}^{2}(x, y) d y d s \leq C|\ln \eta|^{-2(2+h)} \varepsilon^{1 / 2}
$$


the last inequality being a consequence of (5.3). Moreover, coming back to (5.2), we have

$$
\mathbb{P}\left(\Lambda_{\varepsilon, \eta}^{c}(s, y)\right) \leq \frac{1}{\eta^{2}} \mathbb{E}|u(s, y)-u(s \wedge(T-\varepsilon), y)|^{2} \leq \frac{C}{\eta^{2}} \int_{T-\varepsilon}^{s} \int_{0}^{1} G_{s-r}^{2}(y, z) d z d r \leq \frac{C \varepsilon^{1 / 2}}{\eta^{2}}
$$

so that

$$
B_{\varepsilon} \leq \frac{C \varepsilon^{1 / 2}}{\eta^{2}} \int_{T-\varepsilon}^{T} \int_{0}^{1} G_{T-s}^{2}(x, y) d y d s \leq \frac{C \varepsilon}{\eta^{2}}
$$

so that, taking $\eta=\varepsilon^{1 / 16}$ we obtain

$$
\mathbb{E}\left|I_{\varepsilon}(T, x)\right|^{2} \leq C\left(|\ln \varepsilon|^{-2(2+h)}+\varepsilon^{1 / 4}\right) \varepsilon^{1 / 2} \leq C|\ln \varepsilon|^{-2(2+h)} \varepsilon^{1 / 2} .
$$

We estimate now

$$
\left|J_{\varepsilon}(T, x)\right| \leq\|b\|_{\infty} \int_{T-\varepsilon}^{T} \int_{0}^{1} G_{T-s}(x, y) d y d s=\|b\|_{\infty} \varepsilon
$$

so (5.8) is proved.

Step 2. Conditionally to $\mathcal{F}_{T-\varepsilon}$ the random vector $U_{\varepsilon}=\left(u_{\varepsilon}\left(T, x_{1}\right), \ldots, u_{\varepsilon}\left(T, x_{d}\right)\right)$ is Gaussian of covariance matrix

$$
\Sigma^{i, j}\left(U_{\varepsilon}\right)=\int_{T-\varepsilon}^{T} \int_{0}^{1} G_{T-s}\left(x_{i}, y\right) G_{T-s}\left(x_{j}, y\right) \sigma^{2}(u(s \wedge(T-\varepsilon), y)) d y d s, \quad i, j=1, \ldots, d .
$$

By (5.4)

$$
C \sqrt{\varepsilon} \geq \Sigma\left(U_{\varepsilon}\right) \geq \frac{1}{C} \sqrt{\varepsilon}
$$

where $C$ is a constant which depends on the upper bounds of $\sigma$ and on $c_{\sigma}$.

We use now the criterion given in (2.12) with $k=1$ and $q=0$. Let $p_{U_{\varepsilon}}$ be the density of the law of $U_{\varepsilon}$. Conditionally to $\mathcal{F}_{T-\varepsilon}$ this is a Gaussian density and direct estimates give (with the notation from Section 2.1)

$$
\left\|p_{U_{\varepsilon}}\right\|_{2 m, 2 m, e_{\log }} \leq C \varepsilon^{-m / 2} .
$$

So if $\mu_{\varepsilon}$ is the law of $U_{\varepsilon}$ then $\mu_{\varepsilon} \in M_{m, 0, e_{\mathrm{log}}}\left(C \varepsilon^{-m / 2}\right)$. This is true for every $m \in \mathbb{N}$. Having in mind (5.9) and taking $R_{\varepsilon}=C \varepsilon^{-m / 2}$ the quantity in (2.12) is

$$
\begin{aligned}
R_{\varepsilon}^{\frac{1}{2 m}}\left|\ln R_{\varepsilon}\right|^{a\left(1+\frac{1}{2 m}\right)+1} d_{1}\left(\mu, \mu_{\varepsilon}\right) & =C \varepsilon^{-1 / 4}|\ln \varepsilon|^{a\left(1+\frac{1}{2 m}\right)+1} \times|\ln \varepsilon|^{-(2+h)} \varepsilon^{1 / 4} \\
& =C|\ln \varepsilon|^{a\left(1+\frac{1}{2 m}\right)-1-h}
\end{aligned}
$$

where $h>0$ is fixed, being the one in (5.6), and $a>1$ is arbitrarily close to one. Then taking $m$ sufficiently large we upper bound the above term by $C|\ln \varepsilon|^{-h / 2}$ and so we obtain

$$
\varlimsup_{\lim _{\varepsilon \infty 0}} R_{\varepsilon}^{\frac{1}{2 m}}\left|\ln R_{\varepsilon}\right|^{a\left(1+\frac{1}{2 m}\right)+1} d_{1}\left(\mu, M_{m, 0, e_{\log }}\left(R_{\varepsilon}\right)\right)=0 .
$$

Using now the result given in Example 2 we conclude that $\mu$ is absolutely continuous. 


\section{Appendix}

\subsection{Super kernels}

We consider a super kernel $\phi: \mathbb{R}^{d} \rightarrow \mathbb{R}_{+}$that is a function which has the support included in $B_{1}(0), \int \phi(x) d x=1$ and such that for every non null multi index $\alpha=\left(\alpha_{1}, \ldots, \alpha_{d}\right) \in \mathbb{N}^{d}$ one has

$$
\int y^{\alpha} \phi(y) d y=0 \quad y^{\alpha}=\prod_{i=1}^{d} y_{i}^{\alpha_{i}}
$$

See ([15]) Section 3, Remark 1 for the construction of a superkernel. The corresponding $\phi_{\delta}, \delta \in(0,1)$, is defined by

$$
\phi_{\delta}(y)=\frac{1}{\delta^{d}} \phi\left(\frac{y}{\delta}\right)
$$

For a function $f$ we denote $f_{\delta}=f * \phi_{\delta}$. We will work with the norms $\|f\|_{k, \infty}$ and $\|f\|_{q, l,(e)}$ defined in (2.5) and in (2.6). And we have

Lemma 6.1 i) Let $k, q \in \mathbb{N}, l>d$ and $e \in \mathcal{E}$. There exists a universal constant $C$ such that for every $f \in W^{q, l, e}$ one has

$$
\left\|f-f_{\delta}\right\|_{W_{*}^{k, \infty}} \leq C\|f\|_{q, l,(e)} \delta^{q+k} .
$$

ii) Let $k, q \in \mathbb{N}$ and let $e \in \mathcal{E}$. There exists a universal constant $C$ such that for every $f \in W^{q, 0, e}$ one has

$$
\left\|f-f_{\delta}\right\|_{W_{*}^{k, e_{*}}} \leq C\|f\|_{q, 0,(e)} \delta^{q+k} .
$$

iii) Let $l>d, n, q \in \mathbb{N}$, with $n \geq q$, and let $e \in \mathcal{E}$. There exists a universal constant $C$ such that

$$
\left\|f_{\delta}\right\|_{n, l,(e)} \leq C\|f\|_{q, l,(e)} \delta^{-(n-q)}
$$

Proof. i) We may suppose without loss of generality that $f \in C_{b}^{\infty}$. Using Taylor expansion of order $q+k$

$$
\begin{aligned}
f(x)-f_{\delta}(x) & =\int(f(x)-f(y)) \phi_{\delta}(x-y) d y \\
& =\int I(x, y) \phi_{\delta}(x-y) d y+\int R(x, y) \phi_{\delta}(x-y) d y
\end{aligned}
$$

with

$$
\begin{aligned}
I(x, y) & =\sum_{i=1}^{q+k-1} \frac{1}{i !} \sum_{|\alpha|=i} \partial^{\alpha} f(x)(x-y)^{\alpha} \\
R(x, y) & =\frac{1}{(q+k) !} \sum_{|\alpha|=q+k} \int_{0}^{1} \partial^{\alpha} f(x+\lambda(y-x))(x-y)^{\alpha} d \lambda .
\end{aligned}
$$

Using (6.1) we obtain $\int I(x, y) \phi_{\delta}(x-y) d y=0$ and by a change of variable we get

$$
\int R(x, y) \phi_{\delta}(x-y) d y=\frac{1}{(q+k) !} \sum_{|\alpha|=q+k} \int_{0}^{1} \int d z \phi_{\delta}(z) \partial^{\alpha} f(x+\lambda z) z^{\alpha} d \lambda .
$$


We consider now $g \in W^{k, \infty}$ and we write

$$
\int\left(f(x)-f_{\delta}(x)\right) g(x) d x=\frac{1}{(q+k) !} \sum_{|\alpha|=q+k} \int_{0}^{1} d \lambda \int d z \phi_{\delta}(z) z^{\alpha} \int \partial^{\alpha} f(x+\lambda z) g(x) d x .
$$

Let us denote $f_{a}(x)=f(x+a)$. We have $\left(\partial^{\alpha} f\right)(x+a)=\left(\partial^{\alpha} f_{a}\right)(x)$. Let $\alpha$ with $|\alpha|=$ $\sum_{i=1}^{d} \alpha_{i}=q+k$. We split $\alpha$ into two multi indexes $\beta$ and $\gamma$ such that $|\beta|=k,|\gamma|=q$ and $\partial^{\beta} \partial^{\gamma}=\partial^{\alpha}$ (this may be done in several ways but any one of them is good for us). Then using integration by parts

$$
\begin{aligned}
\left|\int \partial^{\alpha} f(x+\lambda z) g(x) d x\right| & =\left|\int \partial^{\beta} \partial^{\gamma} f_{\lambda z}(x) g(x) d x\right| \\
& \leq \int\left|\partial^{\gamma} f_{\lambda z}(x)\right|\left|\partial^{\beta} g(x)\right| d x \leq\|g\|_{k, \infty} \int\left|\partial^{\gamma} f_{\lambda z}(x)\right| d x \\
& =\|g\|_{k, \infty} \int\left|\partial^{\gamma} f(x)\right| d x .
\end{aligned}
$$

We write $\partial^{\gamma} f(x)=u_{l}(x) v_{\gamma}(x)$ with $u_{l}(x)=\left(1+|x|^{2}\right)^{l / 2}$ and $v_{\gamma}(x)=\left(1+|x|^{2}\right)^{-l / 2} \partial^{\alpha} f(x)$. Using Hölder inequality

$$
\int\left|\partial^{\gamma} f(x)\right| d x \leq C\left\|u_{l}\right\|_{\left(e_{*}\right)}\left\|v_{\gamma}\right\|_{(e)} \leq C\left\|u_{l}\right\|_{\left(e_{*}\right)}\|f\|_{q, l,(e)} .
$$

By Remark 2.1 $\left\|u_{l}\right\|_{\left(e_{*}\right)}<\infty$. So we obtain

$$
\begin{aligned}
\left|\int_{0}^{1} \int d z \phi_{\delta}(z) z^{\alpha} \int \partial^{\alpha} f(x+\lambda z) g(x) d x d \lambda\right| & \leq C\|f\|_{q, l,(e)}\|g\|_{k, \infty} \int \phi_{\delta}(z)|z|^{k+q} d z \\
& \leq C\|f\|_{q, l,(e)}\|g\|_{k, \infty} \delta^{k+q} .
\end{aligned}
$$

ii) The proof is exactly the same but one uses directly Hölder's inequality

$$
\int\left|\partial^{\gamma} f_{\lambda z}(x)\right|\left|\partial^{\beta} g(x)\right| d x \leq 2\left\|\partial^{\gamma} f_{\lambda z}\right\|_{(e)}\left\|\partial^{\beta} g\right\|_{\left(e_{*}\right)} .
$$

And we have

$$
\begin{aligned}
\left\|\partial^{\gamma} f_{\lambda z}\right\|_{(e)} & =\inf \left\{c: \int e\left(\frac{1}{c} \partial^{\gamma} f(x+\lambda z)\right) d x \leq 1\right\} \\
& =\inf \left\{c: \int e\left(\frac{1}{c} \partial^{\gamma} f(x)\right) d x \leq 1\right\}=\left\|\partial^{\gamma} f\right\|_{(e)} .
\end{aligned}
$$

iii) Let $\alpha$ be a multi index with $|\alpha|=n$ and let $\beta, \gamma$ be a splitting of $\alpha$ with $|\beta|=q$ and $|\gamma|=n-q$. We have

$$
\begin{aligned}
u(x) & :=(1+|x|)^{l}\left|\partial^{\alpha} f_{\delta}(x)\right|=(1+|x|)^{l}\left|\partial^{\beta} f * \partial^{\gamma} \phi_{\delta}(x)\right| \\
& \leq(1+|x|)^{l}\left|\partial^{\beta} f\right| *\left|\partial^{\gamma} \phi_{\delta}\right|(x) \leq v *\left|\partial^{\gamma} \phi_{\delta}\right|(x)
\end{aligned}
$$


with $v(x)=2^{l}(1+|x|)^{l}\left|\partial^{\beta} f\right|(x)$. The last inequality is due to the fact that if $\left|\partial^{\gamma} \phi_{\delta}\right|(x-$ $y) \neq 0$ then $(1+|y|)^{l} \leq 2(1+|x|)^{l}$. Using (2.17) we obtain

$$
\|u\|_{(e)} \leq\left\|v *\left|\partial^{\gamma} \phi_{\delta}\right|\right\|_{(e)} \leq\left\|\partial^{\gamma} \phi_{\delta}\right\|_{1}\|v\|_{(e)} \leq \frac{C}{\delta^{n-q}}\|v\|_{(e)} \leq \frac{C}{\delta^{n-q}}\left\|f_{\beta, l}\right\|_{(e)} .
$$

Proposition 6.2 Let $r, k, n \in \mathbb{N}$, with $n>r$ and $l>d$, and $e \in \mathcal{E}$. We take $\theta=\frac{k+r}{n-r}$ and $\gamma<\frac{\theta}{1+\theta}=\frac{k+r}{k+n}$. Then (with the notation from Section 3)

$$
W^{r, l, e} \subset B_{\theta, 0}\left(W^{n, l, e}, W_{*}^{k, \infty}\right) \subset\left(W^{n, l, e}, W_{*}^{k, \infty}\right)_{\gamma} .
$$

Proof. We denote $X=W^{n, l, e}, Y=W_{*}^{k, \infty}$. Let $f \in W^{r, l, e}$ and $\delta \in(0,1)$. From (6.4) we have

$$
\left\|f_{\delta}\right\|_{X}=\left\|f_{\delta}\right\|_{n, l,(e)} \leq C\|f\|_{r, l,(e)} \delta^{-(n-r)}=: R .
$$

So $f_{\delta} \in B_{X}(R)$. On the other hand, (6.2) gives

$$
\left\|f-f_{\delta}\right\|_{Y}=\left\|f-f_{\delta}\right\|_{W_{*}^{k, \infty}} \leq C\|f\|_{r, l,(e)} \delta^{k+r}=C\|f\|_{r, l,(e)}^{\rho} R^{-\frac{k+r}{n-r}}
$$

so that

$$
R^{\frac{k+r}{n-r}} d_{Y}\left(f, B_{X}(R)\right) \leq C\|f\|_{r, l,(e)}^{\rho} .
$$

This means that $f \in B_{\theta, 0}(X, Y)$.

We prove now the second inclusion. We have $B_{\theta, 0}(X, Y) \subset B_{\alpha, \beta}(X, Y)$ for every $\alpha<\theta$ and every $\beta \geq 0$. We recall that by Proposition 3.1 we have $B_{\alpha, \beta}(X, Y) \subset(X, Y)_{\gamma}$ with $\alpha=\frac{\gamma}{1-\gamma}$ and $\beta=\frac{2}{1-\gamma}$. By our hypothesis $\alpha=\frac{\gamma}{1-\gamma}<\theta$ so we obtain $B_{\theta, 0}(X, Y) \subset$ $B_{\alpha, \beta}(X, Y) \subset(X, Y)_{\gamma}$.

Recall now Hypothesis $H_{q}(k, m, e)$ and the set $B_{q}(k, m, e)$ defined in Section 2.1. To shorten notations, we put $X=W^{2 m+q, 2 m, e}, Y=W_{*}^{k, \infty}$. Then $M_{m, q, e}(R)=B_{X}(R)$ and $d_{k}\left(\mu, M_{m, q, e}(R)\right)=d_{Y}\left(\mu, B_{X}(R)\right)$. For $a>1$ we have denoted $L_{a}(R)=R(\ln R)^{a}$ and Hypothesis $H_{q}(k, m, e)$ reads: there exists $a>1$ such that

$$
\varlimsup_{R \rightarrow \infty} \frac{L_{a}(R)^{1+\frac{k+q}{2 m}}}{R} \beta_{e}\left(L_{a}(R)^{\frac{d}{2 m}}\right) d_{Y}\left(u, B_{X}(R)\right)<\infty .
$$

And $B_{q}(k, m, e)$ is the set of measures $\mu$ such that $H_{q}(k, m, e)$ holds for $\mu$.

Corollary 6.3 Let $k, q, m \in \mathbb{N}$ with $m>d / 2$, and $e \in \mathcal{E}_{\alpha, \beta}$ (see (2.11)). Suppose that $0 \leq \alpha<\frac{2 m+k+q}{d(2 m-1)}$ and $\beta \geq 0$. Then

$$
W^{q+1,2 m, e} \subset B_{q}(k, m, e) .
$$

Proof. We take $r=q+1, n=2 m+q, l=2 m$ in Proposition 6.2. Then $\theta=\frac{k+q+1}{2 m-1}$. So we know that for $u \in W^{q+1,2 m, e}$ we have

$$
\varlimsup_{R \rightarrow \infty} R^{\frac{k+q+1}{2 m-1}} d_{Y}\left(u, B_{X}(R)\right)<\infty .
$$

Moreover, by (2.11), for sufficiently large $R$

$$
\frac{L_{a}(R)^{1+\frac{k+q}{2 m}}}{R} \beta_{e}\left(L_{a}(R)^{\frac{d}{2 m}}\right) \leq C R^{\frac{k+q}{2 m}+\frac{d \alpha}{2 m}}(\ln R)^{\rho}
$$

with $\rho=a\left(1+\frac{k+q}{2 m}+\frac{d}{2 m}\right)+\beta$. Our hypothesis on $\alpha$ ensures that $\frac{k+q+1}{2 m-1}>\frac{k+q}{2 m}+\frac{d \alpha}{2 m}$ and so (6.7) implies (6.6). 


\subsection{Norms}

In this section we use the notation introduced in Section 3 which we recall here. We consider two normed spaces $\left(X,\|\circ\|_{X}\right)$ and $\left(Y,\|\circ\|_{Y}\right)$ such that $X \subset Y$. We also consider some $a \geq 0, m \in \mathbb{N}_{*}$ and $\theta>0$. For $y \in Y$ and for a sequence $x_{n} \in X, n \in \mathbb{N}$ we define

$$
\pi_{\theta, m, a}\left(y,\left(x_{n}\right)_{n}\right)=\sum_{n=1}^{\infty} 2^{n \theta} n^{a}\left\|y-x_{n}\right\|_{Y}+\frac{1}{2^{2 n m}}\left\|x_{n}\right\|_{X} .
$$

Moreover we define $\rho_{\theta, m, a}^{X, Y}(y)=\inf \pi_{\theta, m, a}(y)$ with the infimum taken over all the sequences $x_{n} \in X, n \in \mathbb{N}$. Finally we denote

$$
S_{\theta, m, a}(X, Y)=\left\{y \in Y: \rho_{\theta, m, a}^{X, Y}(y)<\infty\right\} .
$$

Moreover we denote $K(y, t)=\inf \left\{\|y-x\|_{Y}+t\|x\|_{X}\right\}$ with the infimum taken over all $x \in X$ and we define

$$
|y|_{\gamma, b}=\int_{0}^{1} \frac{|\ln t|^{b}}{t^{\gamma}} K(y, t) \frac{d t}{t}
$$

We denote

$$
K_{\gamma, b}(X, Y)=\left\{y \in Y:|y|_{\gamma, b}<\infty\right\}
$$

Proposition 6.4 We have

$$
S_{\theta, m, a}(X, Y)=K_{\gamma, b}(X, Y) \quad \text { with } \quad \gamma=\frac{\theta}{2 m+\theta}, b=\frac{2 m a}{2 m+\theta} .
$$

and there exists a universal constant $C$ (which may be computed explicitly) such that

$$
\frac{1}{C} \rho_{\theta, m, a}^{X, Y}(y) \leq|y|_{\gamma, b} \leq C\left(\|y\|_{Y}+\rho_{\theta, m, a}^{X, Y}(y)\right)
$$

Proof. Step 1. We write

$$
\pi_{\theta, m, a}\left(y,\left(x_{n}\right)_{n}\right)=\sum_{n} 2^{n \theta} n^{a}\left(\left\|y-x_{n}\right\|_{Y}+\frac{1}{n^{a} 2^{n(2 m+\theta)}}\left\|x_{n}\right\|_{X}\right)
$$

and we define

We have

$$
t_{n}=\frac{1}{n^{a} 2^{n(2 m+\theta)}} .
$$

$$
t_{n}-t_{n+1}=t_{n} \alpha_{n} \quad \text { with } \quad \alpha_{n}=1-\frac{1}{2^{2 m+\theta}} \times\left(\frac{n}{n+1}\right)^{a} .
$$

And

$$
\alpha^{*}:=1-\frac{1}{2^{2 m+\theta+a}} \geq \alpha_{n} \geq 1-\frac{1}{2^{2 m+\theta}}=: \alpha_{*} .
$$

Then we write

$$
\pi_{\theta, m, a}\left(y,\left(x_{n}\right)_{n}\right)=\sum_{n} 2^{n \theta} n^{a} \times \frac{\left\|y-x_{n}\right\|_{Y}+t_{n}\left\|x_{n}\right\|_{X}}{t_{n} \alpha_{n}} \times\left(t_{n}-t_{n+1}\right)
$$


so that

$$
\frac{1}{\alpha^{*}} \pi_{\theta, m, a}^{\prime}\left(y,\left(x_{n}\right)_{n}\right) \leq \pi_{\theta, m, a}\left(y,\left(x_{n}\right)_{n}\right) \leq \frac{1}{\alpha_{*}} \pi_{\theta, m, a}^{\prime}\left(y,\left(x_{n}\right)_{n}\right)
$$

with

$$
\pi_{\theta, m, a}^{\prime}\left(y,\left(x_{n}\right)_{n}\right)=\sum_{n} 2^{n \theta} n^{a} \times \frac{\left\|y-x_{n}\right\|_{Y}+t_{n}\left\|x_{n}\right\|_{X}}{t_{n}} \times\left(t_{n}-t_{n+1}\right) .
$$

Step 2. We have

$$
\frac{\left|\ln t_{n}\right|^{b}}{t_{n}^{\theta /(2 m+\theta)}}=(a \ln n+n(2 m+\theta) \ln 2)^{b} n^{a \theta /(2 m+\theta)} 2^{n \theta}
$$

Since $b+a \theta /(2 m+\theta)=a$ we may find $C$ such that

$$
C \times n^{a} 2^{n \theta} \geq \frac{\left|\ln t_{n}\right|^{b}}{t_{n}^{\theta /(2 m+\theta)}} \geq \frac{1}{C} \times n^{a} 2^{n \theta}
$$

Notice that the functions $t \rightarrow t^{-1} K(y, t)$ and $t \rightarrow t^{-1} y /(2 m+\theta)|\ln t|^{b}$ are decreasing so we obtain

$$
\frac{\left|\ln t_{n+1}\right|^{b}}{t_{n+1}^{\theta /(2 m+\theta)}} \frac{K\left(y, t_{n+1}\right)}{t_{n+1}}\left(t_{n}-t_{n+1}\right) \geq \int_{t_{n+1}}^{t_{n}} \frac{|\ln t|^{b}}{t^{\theta /(2 m+\theta)}} K(y, t) \frac{d t}{t} \geq \frac{\left|\ln t_{n}\right|^{b}}{t_{n}^{\theta /(2 m+\theta)}} \frac{K\left(y, t_{n}\right)}{t_{n}}\left(t_{n}-t_{n+1}\right) .
$$

By the very definition of $K\left(y, t_{n}\right)$ we may find $x_{n} \in X$ such that $K\left(y, t_{n}\right) \geq \frac{1}{2}\left(\left\|y-x_{n}\right\|_{Y}+\right.$ $\left.t_{n}\left\|x_{n}\right\|_{X}\right)$. It follows that

$$
\begin{aligned}
\int_{0}^{1} \frac{|\ln t|^{b}}{t^{\theta /(2 m+\theta)}} K(y, t) \frac{d t}{t} & \geq \sum_{n} \int_{t_{n+1}}^{t_{n}} \frac{|\ln t|^{b}}{t^{\theta /(2 m+\theta)}} K(y, t) \frac{d t}{t} \\
& \geq \frac{1}{2} \sum_{n} \frac{\left|\ln t_{n}\right|^{b}}{t_{n}^{\theta /(2 m+\theta)}} \frac{\left\|y-x_{n}\right\|_{Y}+t_{n}\left\|x_{n}\right\|_{X}}{t_{n}}\left(t_{n}-t_{n+1}\right) \\
& \geq \frac{1}{2 C} \pi_{\theta, m, a}^{\prime}\left(y,\left(x_{n}\right)_{n}\right)
\end{aligned}
$$

So we have proved that for $\gamma=\theta /(2 m+\theta)$ and $b=2 m a /(2 m+\theta)$ one has

$$
|y|_{\gamma, b} \geq \frac{\alpha_{*}}{2 C} \pi_{\theta, m, a}\left(y,\left(x_{n}\right)_{n}\right) \geq \frac{\alpha_{*}}{2 C} \rho_{\theta, m, a}^{X, Y}(y) .
$$

We write now

$$
\begin{aligned}
t_{n}-t_{n+1} & =\alpha_{n} t_{n}=\frac{\alpha_{n}}{\alpha_{n+1}} \times \frac{t_{n}}{t_{n+1}} \times \alpha_{n+1} t_{n+1}=\frac{\alpha_{n}}{\alpha_{n+1}} \times \frac{t_{n}}{t_{n+1}} \times\left(t_{n+1}-t_{n+2}\right) \\
& \leq 2^{2 m+\theta+a+1}\left(t_{n+1}-t_{n+2}\right) .
\end{aligned}
$$

Then

$$
2^{2 m+\theta+a+1} \times \frac{\left|\ln t_{n+1}\right|^{b}}{t_{n+1}^{\theta /(2+\theta)}} \frac{K\left(y, t_{n+1}\right)}{t_{n+1}}\left(t_{n+1}-t_{n+2}\right) \geq \int_{t_{n+1}}^{t_{n}} \frac{|\ln t|^{b}}{t^{\theta /(2+\theta)}} K(y, t) \frac{d t}{t}
$$


For every $x_{n+1} \in X$ we have $K\left(y, t_{n+1}\right) \leq\left\|y-x_{n+1}\right\|_{Y}+t_{n+1}\left\|x_{n+1}\right\|_{X}$ so that for every sequence $x_{n} \in X$ we obtain

$$
\begin{aligned}
2^{2 m+\theta+a+1} & \times \sum_{n} \frac{\left|\ln t_{n+1}\right|^{b}}{t_{n+1}^{\theta /(2+\theta)}} \frac{\left\|y-x_{n+1}\right\|_{Y}+t_{n+1}\left\|x_{n+1}\right\|_{X}}{t_{n+1}}\left(t_{n+1}-t_{n+2}\right) \geq \\
& \geq \sum_{n} \int_{t_{n+1}}^{t_{n}} \frac{|\ln t|^{b}}{t^{\theta /(2+\theta)}} K(y, t) \frac{d t}{t} .
\end{aligned}
$$

This means that

$$
2^{2 m+\theta+a+1} \alpha^{*} \pi_{\theta, m, a}\left(y,\left(x_{n}\right)_{n}\right) \geq 2^{2 m+\theta+a+1} \pi_{\theta, m, a}^{\prime}\left(y,\left(x_{n}\right)_{n}\right) \geq \int_{0}^{t_{1}} \frac{|\ln t|^{b}}{t^{\theta /(2+\theta)}} K(y, t) \frac{d t}{t} .
$$

Since this inequality holds for every sequence it holds for the infimum also. So we obtain

$$
2^{2 m+\theta+a+1} \alpha^{*} \rho_{\theta, m, a}^{X, Y}(y) \geq \int_{0}^{t_{1}} \frac{|\ln t|^{b}}{t^{\theta /(2+\theta)}} K(y, t) \frac{d t}{t},
$$

and the statement follows.

We define now

$$
B_{\alpha, \beta}(X, Y)=\left\{y \in Y: \varlimsup_{R \rightarrow \infty} R^{\alpha}(\ln R)^{\beta} d_{Y}\left(y, B_{X}(R)\right)<\infty\right\}
$$

\section{Proposition 6.5}

$$
B_{\alpha, \beta}(X, Y) \subset S_{\theta, m, a}(X, Y) \quad \text { with } \quad \alpha=\frac{\theta}{2 m}, \beta=2+a+\frac{\theta}{m} .
$$

Proof. If $y \in B_{\alpha, \beta}(X, Y)$ one may find $R_{*}, C_{*}$ such that for every $R \geq R_{*}$ there exists $x_{R} \in X$ such that

$$
\left\|x_{R}\right\|_{X} \leq R \quad \text { and } \quad\left\|y-x_{R}\right\|_{X} \leq \frac{C_{*}}{R^{\alpha}(\ln R)^{\beta}} .
$$

We take $R_{n}=n^{-2} 2^{2 n m}$ and $n_{*}$ such that $R_{n_{*}} \geq R_{*}$. Then

$$
\sum_{n \geq n_{*}} \frac{1}{2^{2 n m}}\left\|x_{R n}\right\|_{X} \leq \sum_{n \geq n_{*}} \frac{1}{n^{2}}<\infty .
$$

Moreover, since $2 m \alpha=\theta$ and $\beta-a-2 \alpha=2$ we have

$$
n^{a} 2^{n \theta} \times \frac{1}{R_{n}^{\alpha}\left(\ln R_{n}\right)^{\beta}}=n^{a} 2^{n \theta} \times \frac{n^{2 \alpha}}{2^{2 n m \alpha}(2 n m \ln 2-2 \ln n)^{\beta}} \leq \frac{C}{n^{\beta-a-2 \alpha}}=\frac{C}{n^{2}}
$$

so that

$$
\sum_{n \geq n_{*}} n^{a} 2^{n \theta}\left\|y-x_{R_{n}}\right\|_{X} \leq \sum_{n \geq n_{*}} \frac{1}{n^{2}}<\infty
$$


We come now back to the "balance" discussed in Section 2.1. We recall that for $a>1$ we have denoted $L_{a}(R)=R(\ln R)^{a}$. We also considered a general Young function $e \in \mathcal{E}$ and we gave the hypothesis $H_{q}(k, m, e)$. We give here the statement of this hypothesis in our abstract setting. Let $\theta \geq 0$ and $y \in Y$.

Hypothesis $H(\theta, m, e)$. For $\theta \in \mathbb{N}, m \in \mathbb{N}_{*}$ and $e \in \mathcal{E}$ there exists $a>1$ such that

$$
\varlimsup_{R \rightarrow \infty} \frac{L_{a}(R)^{1+\frac{\theta}{2 m}}}{R} \beta_{e}\left(L_{a}(R)^{d / 2 m}\right) d_{Y}\left(y, B_{X}(R)\right)<\infty
$$

For $\theta=k+q, X=W^{2 m+q, 2 m, e}$ and $Y=W_{*}^{k, \infty}$ we obtain $H_{q}(k, m, e)$.

Lemma 6.6 If $H(\theta, m, e)$ holds for $y \in Y$ then one may find a sequence $x_{n}$ such that

$$
\sum_{n=1}^{\infty} 2^{n \theta} \beta_{e}\left(2^{n d}\right)\left\|y-x_{n}\right\|_{Y}+\frac{1}{2^{2 n m}}\left\|x_{n}\right\|_{X}<\infty .
$$

Proof. For a suitable $a>1$ and large $R$ we have

$$
d_{Y}\left(\mu, B_{X}(R)\right) \leq \frac{C R}{L_{a}(R)^{1+\frac{\theta}{2 m}} \beta_{e}\left(L_{a}(R)^{d / 2 m}\right)} .
$$

We choose $R_{n}=n^{-a} 2^{2 n m}$ and we take a sequence $x_{n} \in B_{X}\left(R_{n}\right)$ such that

$$
\left\|y-x_{n}\right\|_{Y} \leq \frac{C R_{n}}{L_{a}\left(R_{n}\right)^{1+\frac{\theta}{2 m}} \beta_{e}\left(L_{a}\left(R_{n}\right)^{d / 2 m}\right)} .
$$

By the very definition of $B_{X}\left(R_{n}\right)$ we have

$$
\sum_{n=0}^{\infty} \frac{1}{2^{2 n m}}\left\|x_{n}\right\|_{X} \leq \sum_{n=0}^{\infty} \frac{1}{2^{2 n m}} R_{n} \leq \sum_{n=0}^{\infty} \frac{1}{n^{a}}<\infty .
$$

One also has

$$
(2 m)^{a} \times 2^{2 n m} \geq L_{a}\left(R_{n}\right)=\frac{1}{n^{a}} 2^{2 n m}(2 n m \ln 2-a \ln n)^{a} \geq 2^{2 n m}
$$

the last inequality being true for sufficiently large $n$ (we need $2 n m \ln 2-a \ln n \geq n$ ). It follows that $L_{a}\left(R_{n}\right)^{d / 2 m} \geq 2^{\text {nd }}$ and this yields $\beta_{e}\left(L_{a}\left(R_{n}\right)^{d / 2 m}\right) \geq \beta_{e}\left(2^{n d}\right)$. We conclude that

$$
\begin{aligned}
2^{n \theta} \beta_{e}\left(2^{n d}\right)\left\|y-x_{n}\right\|_{Y} & \leq 2^{n \theta} \beta_{e}\left(2^{n d}\right) \times \frac{C}{L_{a}\left(R_{n}\right)^{\frac{\theta}{2 m}} \beta\left(L_{a}\left(R_{n}\right)^{d / 2 m}\right)} \times \frac{R_{n}}{L_{a}\left(R_{n}\right)} \\
& \leq(2 m)^{a} \times \frac{C}{\left(\ln R_{n}\right)^{a}} \leq \frac{C(2 m)^{a}}{n^{a}}
\end{aligned}
$$

which shows that the first series is also convergent. 


\section{References}

[1] V. Bally, L. Caramellino (2011). Riesz transform and integration by parts formulas for random variables. Stochastic Process. Appl., 121, 1332-1355.

[2] V. Bally, E. Clément (2011). Integration by parts formulas and applications to equations with jumps. PTRF 151, 613-657.

[3] V. BAlly, E. Clément (2011). Integration by parts formulas with respect to jump times and stochastic differential equations. Stochastic Analysis 2010, ed. Dan Crisan, Springer.

[4] V. Bally, N. Fournier (2011). Regularization properties od the 2D homogeneous Boltzmann equation without cutoff. PTRF 151, 659-704.

[5] V. Bally, E. Pardoux (1998). Malliavin Calculus for White Noise Driven Parabolic SPDE's. Potential Analysis 9, 27-64.

[6] C. Bennett, R. Sharpley (1988). Interpolation of operators. Academic Press INC.

[7] P. Cattiaux (1986). Hypoellipticité et hypoellipticité partielle pour les diffusions avec une condition frontiere. Ann. Inst. H. Poincaré Probab. Stat. 22, 67-112.

[8] A. Debussche, M. Romito (2012). Existence of densities for the 3D Navier-Stokes equations driven by Gaussian noise, Preprint arXiv:1203.0417.

[9] S. De Marco (2011). Smoothness and Asymptotic Estimates of densities for SDEs with locally smooth coefficients and Applications to square-root diffusions. The Annals of Applied Probability 21, 1282-1321.

[10] J. DziUbanski (1997). Triebel-Lizorkin spaces associated with Laguerre and Hermite expansions. Proc. Amer. Math. Soc. 125, 3547-3554.

[11] J. Epperson (1985). Hermite and Laguerre wave packet expansions. Studia Math. J. 34, 777-799.

[12] N. Fournier (2008). Smoothness of the law of some one-dimensional jumping SDE's with non constant rate of jump. Electron. J. Probab. 13, 135-156.

[13] N. Fournier (2012). Finiteness of entropy for the homogeneous Boltzmann equation with measure initial condition. Preprint arXiv 1203.0130.

[14] N. Fournier, J. Printems (2010). Absolute continuity of some one-dimensional processes. Bernoulli 16, 343-360.

[15] A. Kebaier, A. Kohatsu-Higa (2008). An optimal control variance reduction method for density estimation. Stochastic Process. Appl. 118, 2143-2180. 
[16] P. Kosmol, D. Mûller-Wichards (2011). Optimization in Functional Spaces with stability considerations in Orlicz spaces. De Gruyter Series in Non Linear Analysis and Applications 13. Walter de Gruyter.

[17] S. Kusuoka, D. Stroock (1987). Applications of the Malliavin calculus. III. J. Fac. Sci. Univ. Tokyo Sect. IA Math., 34, 391-442.

[18] P. Malliavin (1997). Stochastic Analysis. Springer.

[19] P. Malliavin, A. Thalmaier (2006). Stochastic calculus of variations in mathematical finance. Springer-Verlag, Berlin.

[20] S. Ninomiya, N. Victoir (2008). Weak approximation of stochastic differential equations and applications to derivative pricing. Appl. Math. Finance, 15, 107-121.

[21] D. Nualart (2006) The Malliavin calculus and related topics. Second Edition. Springer-Verlag.

[22] P. Petrushev, Yuan Xu (2008). Decomposition of spaces of distributions induced by Hermite expansions. J. Fourier Anal. and Appl. 14, 372-414.

[23] E. Pardoux, T. Zhang (1993). Absolute continuity for the law of the solution of a parabolic SPDE. J. Funct. Anal. 112, 447-458.

[24] M. Sanz Sol̀̀ (2005). Malliavin Calculus, with Applications to Stochastic Partial Differential Equations. EPFL Press. Fundamental Sciences, Mathematics.

[25] I. Shigekawa (2004). Stochastic analysis. In: Translations of Mathematical Monographs, in: Iwanami Series in Modern Mathematics, vol. 224, American Mathematical Society, Providence.

[26] D. Talay, L. Tubaro (1990). Expansion of the global error for numerical schemes solving stochastic differential equations. Stochastica Analysis and Applications, 8, 94-120.

[27] H. Triebel (1999). Interpolation Theory - Function Spaces - Differential Operators. John Wiley \& Sons, Incorporated.

[28] J.B. WAlsh (1986). An introduction to stochastic partial differential equations. In Ecole d'Eté de Probabilités de Saint Flour XV, Lecture Notes in Math. 1180, Springer; pp 226-437. 